\title{
ELLIPTIC AND PARABOLIC EQUATIONS WITH MEASURABLE COEFFICIENTS IN $L_{P}$-SPACES WITH MIXED NORMS*
}

\author{
DOYOON $\mathrm{KIM}^{\dagger}$
}

\begin{abstract}
The unique solvability results for second order parabolic and elliptic equations in Sobolev spaces with mixed norms are presented. The second order coefficients are measurable in one spatial variable and VMO (vanishing mean oscillation) in the other spatial variables. In the parabolic case, the coefficients (except $a^{11}$ ) are further allowed to be only measurable in time. We first prove the solvability results for equations in the whole Euclidean space. Then, using these results as well as some extension techniques, we prove the solvability results for equations on a half space without any boundary estimates. The mixed norms we present here are more general than the usual mixed $\operatorname{norm} L_{q}^{t} L_{p}^{x}$.
\end{abstract}

Key words. Second order elliptic and parabolic equations, vanishing mean oscillation, Sobolev spaces with mixed norms.

AMS subject classifications. 35J15, 35K10, 35R05, 35A05

1. Introduction. In this paper we study elliptic and parabolic equations of nondivergence type in Sobolev spaces with mixed norms. The differential equations we consider are (elliptic and parabolic, respectively)

$$
\begin{gathered}
a^{i j}(x) u_{x^{i} x^{j}}+b^{i}(x) u_{x^{i}}+c(x) u=f, \\
u_{t}+a^{i j}(t, x) u_{x^{i} x^{j}}+b^{i}(t, x) u_{x^{i}}+c(t, x) u=f .
\end{gathered}
$$

The equations are assumed to be uniformly non-degenerate with bounded coefficients. The regularity assumptions on the coefficients $a^{i j}$ (no regularity assumptions are needed for coefficients $b^{i}$ and $c$ ) are, roughly speaking, that they are merely measurable (i.e., no regularity assumptions) in one spatial direction, and belong to the space of VMO (mean vanishing oscillation) as functions of the other variables. In the parabolic case, they (except $a^{11}$ ) are measurable in two variables including the time variable and in the space of VMO as functions of the remaining variables. The coefficient $a^{11}$ is measurable in one spatial variable and $\mathrm{VMO}$ in the other variables.

There has been, in fact, considerable study of parabolic equations in mixed norm spaces in the literature (see, e.g., $[4,18,11,10,12,21,3,6,20,14]$ and references therein). The usual mixed norms are of the form $L_{q}\left((0, T), L_{p}\right)$, that is, $q$ summability in the time variable and $p$ summability in the spatial variables. For example, in $[12$, $21,20,14]$ parabolic equations as in (2) (quasi-linear equations in [20]) are investigated in Sobolev spaces with the mixed norm $L_{q}\left((0, T), L_{p}(\Omega)\right)$, where $\Omega \subseteq \mathbb{R}^{d}$. In [3] one sees parabolic systems in $L_{q}((0, T), X)$, where $X$ is an $L_{p}$ space with a Muckenhoupt weight. In this paper, however, by mixed norms we mean, not the usual mixed norms, but more general ones defined as follows.

First we explain some notations. By $\mathbb{R}^{d}$ we mean a $d$-dimensional Euclidean space and a point in $\mathbb{R}^{d}$ is denoted by $x$. In the parabolic case we consider $\mathbb{R}^{d+1}$, where a point in $\mathbb{R}^{d+1}$ is denoted by $(t, x)$. Throughout the paper, we fix two nonnegative

\footnotetext{
*Received October 29, 2008; accepted for publication November 7, 2008.

${ }^{\dagger}$ Department of Mathematics, University of Southern California, 3620 Vermont Avenue, KAP108 Los Angeles, CA 90089, USA (doyoonki@usc.edu).
} 
integers $d_{1}$ and $d_{2}$ such that $d_{1}+d_{2}=d$. We also fix all different integers $i_{1}, i_{2}, \cdots, i_{d_{1}}$ and $j_{1}, j_{2}, \cdots, j_{d_{2}}$ from $\{1,2, \cdots, d\}$. Then for $x=\left(x^{1}, x^{2}, \cdots, x^{d}\right) \in \mathbb{R}^{d}$, we denote

$$
\mathbf{x}_{1}=\left(x^{i_{1}}, x^{i_{2}}, \cdots, x^{i_{d_{1}}}\right) \in \mathbb{R}^{d_{1}}, \quad \mathbf{x}_{2}=\left(x^{j_{1}}, x^{j_{2}}, \cdots, x^{j_{d_{2}}}\right) \in \mathbb{R}^{d_{2}} .
$$

Note that, as one can see, for example, $\mathbf{x}_{1}$ consists of $d_{1}$ coordinates, but $\boldsymbol{x}_{1}$ is not necessarily the first $d_{1}$ coordinates of $x \in \mathbb{R}^{d}$. Likewise, $\mathbb{R}^{d_{1}}$ above is a subspace of $\mathbb{R}^{d}$, but not necessarily consists of the first $d_{1}$ coordinates of points in $\mathbb{R}^{d}$. If $\mathcal{D}$ is a subset in $\mathbb{R}^{d+1}$, we define

$$
\|u\|_{L_{q}^{t, \mathbf{x}_{1}} L_{p}^{\mathbf{x}_{2}}(\mathcal{D})}=\left(\int_{\mathbb{R} \times \mathbb{R}^{d_{1}}}\left(\int_{\mathbb{R}^{d_{2}}}|u(t, x)|^{p} I_{\mathcal{D}}(t, x) d \mathbf{x}_{2}\right)^{q / p} d \mathbf{x}_{1} d t\right)^{1 / q},
$$

where $I_{\mathcal{D}}$ is the indicator function. Similarly,

$$
\|u\|_{L_{q}^{x_{1}} L_{p}^{t, \mathbf{x}_{2}}(\mathcal{D})}=\left(\int_{\mathbb{R}^{d_{1}}}\left(\int_{\mathbb{R} \times \mathbb{R}^{d_{2}}}|u(t, x)|^{p} I_{\mathcal{D}}(t, x) d \mathbf{x}_{2} d t\right)^{q / p} d \mathbf{x}_{1}\right)^{1 / q} .
$$

For elliptic equations, we define

$$
\|u\|_{L_{q}^{x_{1}} L_{p}^{x_{2}}(\Omega)}=\left(\int_{\mathbb{R}^{d_{1}}}\left(\int_{\mathbb{R}^{d_{2}}}|u(x)|^{p} I_{\Omega}(x) d \mathbf{x}_{2}\right)^{q / p} \mathbf{x}_{1}\right)^{1 / q},
$$

where $\Omega$ is a subset in $\mathbb{R}^{d}$. Finally we set

$$
\begin{gathered}
L_{q, p}(\mathcal{D}):=L_{q}^{t, \mathbf{x}_{1}} L_{p}^{\mathbf{x}_{2}}(\mathcal{D}) \quad \text { or } \quad L_{q}^{\mathbf{x}_{1}} L_{p}^{t, \mathbf{x}_{2}}(\mathcal{D}), \\
L_{q, p}(\Omega):=L_{q}^{\mathbf{x}_{1}} L_{p}^{\mathbf{x}_{2}}(\Omega) .
\end{gathered}
$$

Note that, in case $p=q, L_{p}(\mathcal{D})=L_{p, p}(\mathcal{D})=L_{p}^{t, \mathbf{x}_{1}} L_{p}^{\mathbf{x}_{2}}(\mathcal{D})=L_{p}^{\mathbf{x}_{1}} L_{p}^{t, \mathbf{x}_{2}}(\mathcal{D})$ and $L_{p}(\Omega)=$ $L_{p, p}(\Omega)$.

Apparently our mixed norms are more general than the usual mixed norms such as $L_{q}\left((0, T), L_{p}\left(\mathbb{R}^{d}\right)\right)$. According to the notation above, $L_{q}\left((0, T), L_{p}\left(\mathbb{R}^{d}\right)\right)$ is denoted by $L_{q}^{t} L_{p}^{x}\left((0, T) \times \mathbb{R}^{d}\right)$. In our case we have $q$ summability in any given variables, which may or may not include the time variable, and $p$ summability in the remaining variables.

With the above mixed norms in hand, we prove that, for a given $f \in L_{q, p}((0, T) \times$ $\left.\mathbb{R}^{d}\right), q>p \geq 2$, there exists a unique solution $u \in W_{q, p}^{1,2}\left((0, T) \times \mathbb{R}^{d}\right)$ to the parabolic equation (2) with an appropriate initial condition, where $u \in W_{q, p}^{1,2}\left((0, T) \times \mathbb{R}^{d}\right)$ means that $u, u_{t}, u_{x^{i}}, u_{x^{i} x^{j}} \in L_{q, p}\left((0, T) \times \mathbb{R}^{d}\right)$. We also prove the unique solvability of the elliptic equation (1) in Sobolev spaces with mixed norms, which are somewhat more general than the usual homogenous Sobolev spaces (no mixed norms) for elliptic equations as in $[5,13,8]$. Considering elliptic equations in mixed norm spaces as ours must be another expansion of $L_{p}$-theory for elliptic equations. The proof for the elliptic case is rather simple and uses the solvability result for parabolic equations.

As explained in [14], the necessity of mixed norms for differential equations arises when one has to improve the regularity of a solution in some directions. For example, if the derivatives of a solution are in $L_{q}^{t} L_{p}^{x}\left((0, T) \times \mathbb{R}^{d}\right)$ and $q$ is large enough, then using embedding theorems we might be able to increase the regularity of the solution in the 
time direction. Indeed, in [7] the solvability of parabolic equations in Sobolev space with mixed norms is used when proving results for equations in Sobolev spaces without mixed norms (for instance, the Hölder continuity of solutions to parabolic equations in Sobolev spaces without mixed norms). Moreover, by having mixed norms as ours we are able to have better regularity of solutions as functions of some given variables (not as functions of only time or only the whole spatial variables). An example of this argument is presented in the proof of Lemma 5.5 of this paper.

We also prove the existence and uniqueness of solutions to equations defined on a half space $\mathbb{R}_{+}^{d}=\left\{x=\left(x^{1}, \cdots, x_{d}\right) \in \mathbb{R}^{d}, x^{1}>0\right\}$. Traditionally, in order to solve equations on a half space or on a bounded domain, one has to have boundary estimates. However, we do not need any boundary estimates but the results for equations in the whole space. As is seen in the proof of Theorem 2.5, this is made possible by our assumption that $a^{i j}$ are measurable in one spatial direction. This shows that the class of coefficients $a^{i j}$ in this paper is very useful. Certainly, our results for equations on a half space can be used, via a partition of unity and the usual flattening argument, when dealing with equations on a bounded domain if the leading coefficients are, for instance, in the class of VMO as functions of $(t, x)$.

The same (or similar) class of coefficients is presented in $[8,9,7]$, where homogeneous Sobolev spaces are considered. Before the current class of coefficients $a^{i j}$ was introduced, one considerably general assumption on $a^{i j}$ had been that they are in the space of VMO, which were first investigated in $[2,1]$. Then recently a new class of coefficients (called $V M O_{x}$ coefficients) was suggested by N. Krylov in [13]. The $V M O_{x}$ coefficients are characterized as being measurable in the time variable and VMO in the spatial variables. For more information about general elliptic and parabolic equations in Sobolev spaces, see $[5,17,19,15]$ and references therein. On the other hand, for example, in $[6,12,14]$ one can see VMO or measurable coefficients for parabolic equations in mixed norm spaces. The coefficients in [6] are VMO in $x \in \mathbb{R}^{d}$, but independent of $t \in \mathbb{R}$, whereas the coefficients $a^{i j}$ in [12] are measurable functions of only $t$, and the coefficients $a^{i j}$ in [14] are $V M O_{x}$ coefficients, the same class as in [13]. Since in our case (in the parabolic case) the coefficients $a^{i j}(t, x)$ (except $\left.a^{11}\right)$ are measurable as functions of $\left(t, x^{1}\right)$ and VMO as functions of $x^{\prime}$, where $x^{\prime}=\left(x^{2}, \cdots, x^{d}\right) \in \mathbb{R}^{d-1}$ ( $a^{11}$ is measurable in $x^{1}$ and VMO in $\left(t, x^{\prime}\right)$ ), as far as coefficients $a^{i j}, i \neq 1$ or $j \neq 1$, are concerned, the class of coefficients in this paper is bigger than those in $[6,12,14]$.

Our approach to dealing with mixed norms is based on the method presented in [14]. In order to have, for example, an $L_{q}^{t, \mathbf{x}_{1}} L_{p}^{\mathbf{x}_{2}}\left(\mathbb{R}^{d+1}\right)$-estimate, we first prove pointwise estimates of the sharp functions of $\left\|u_{x^{i} x^{j}}\left(t, \mathbf{x}_{1}, \cdot\right)\right\|_{L_{p}\left(\mathbb{R}^{\left.d_{2}\right)}\right.}$ as functions of $\left(t, \mathbf{x}_{1}\right) \in \mathbb{R} \times \mathbb{R}^{d_{1}}$. Then we obtain the desired mixed norm estimate using the HardyLittlewood theorem and Fefferman-Stein theorem. This approach is quite different from ones used in many papers about equations in Sobolev spaces with or without mixed norms. Especially, semigroup or singular integral based methods seem not to be applicable in our situation.

This paper consist of two parts. In the first part we solve the equation (2) in Sobolev spaces with mixed norms when the coefficients $a^{i j}$ are measurable in $x^{1} \in \mathbb{R}$ and VMO in $\left(t, x^{\prime}\right) \in \mathbb{R} \times \mathbb{R}^{d-1}$. This result serves as one of main steps in [7]. Then using the results in [7] as well as in the first part of this paper, we prove the main results of this paper. The first part consists of section 3 and 4; the second part consists of section 5 and 6 . In section 2 we states the assumptions and the main results.

A few more words about notation: We denote $x^{\prime}$ the last $d-1$ coordinates of $x$, 
that is, $x^{\prime}=\left(x^{2}, \cdots, x^{d}\right) \in \mathbb{R}^{d-1}$, so that $x=\left(x^{1}, x^{\prime}\right)$. By $u_{x^{\prime}}$ we mean, depending on the context, one of $u_{x^{j}}, i=2, \cdots, d$, or the whole collection $\left\{u_{x^{2}}, \cdots, u_{x^{d}}\right\}$. As usual, $u_{x}$ represents one of $u_{x^{i}}, i=1, \cdots, d$, or the whole collection of $\left\{u_{x^{1}}, \cdots, u_{x^{d}}\right\}$. Thus $u_{x x^{\prime}}$ is one of $u_{x^{i} x^{j}}$, where $i \in\{1, \cdots, d\}$ and $j \in\{2, \cdots, d\}$, or the collection of them. For a function $g$ defined on $\mathbb{R}^{m}$ (or on a subset in $\mathbb{R}^{m}$ ), $m \leq d+1$, the average of $g$ over an open set $\Omega \subset \mathbb{R}^{m}$ is denoted by $(u)_{\Omega}$, i.e.,

$$
(g)_{\Omega}=\frac{1}{|\Omega|} \int_{\Omega} g(x) d x=f_{\Omega} g(x) d x,
$$

where $|\Omega|$ is the $m$-dimensional volume of $\Omega$. Finally, various constants are denoted by $N$, their values may vary from one occurrence to another. We write $N(d, \delta, \ldots)$ if $N$ depends only on $d, \delta, \ldots$.

2. Main results. We consider the elliptic (1) and parabolic equation (2) with coefficients $a^{i j}, b^{i}$, and $c$ satisfying the following assumptions. If the elliptic equation is considered, we assume that all coefficients are independent of $t \in \mathbb{R}$.

Assumption 2.1. The coefficients $a^{i j}, b^{i}$, and $c$ are measurable functions defined on $\mathbb{R}^{d+1}, a^{i j}=a^{j i}$. There exist constants $\delta \in(0,1)$ and $K>0$ such that

$$
\begin{gathered}
\left|b^{i}(t, x)\right| \leq K, \quad|c(t, x)| \leq K, \\
\delta|\vartheta|^{2} \leq \sum_{i, j=1}^{d} a^{i j}(t, x) \vartheta^{i} \vartheta^{j} \leq \delta^{-1}|\vartheta|^{2}
\end{gathered}
$$

for any $(t, x) \in \mathbb{R}^{d+1}$ and $\vartheta \in \mathbb{R}^{d}$.

Another assumption on the coefficients $a^{i j}(t, x)$ is that they are, in case $p \in(2, \infty)$, measurable in $\left(t, x^{1}\right) \in \mathbb{R}^{2}$ and VMO in $x^{\prime}=\left(x^{2}, \cdots, x^{d}\right) \in \mathbb{R}^{d-1}$ (the coefficient $a^{11}(t, x)$ is measurable in $x^{1} \in \mathbb{R}$ and VMO in $\left.\left(t, x^{\prime}\right) \in \mathbb{R}^{d}\right)$. In case $p=2$, the coefficients $a^{i j}(t, x)$ are measurable functions of only $\left(t, x^{1}\right) \in \mathbb{R}^{2}$, but $a^{11}\left(t, x^{1}\right)$ is $\mathrm{VMO}$ in $t \in \mathbb{R}$. If $a^{i j}$ are independent of $t$ (i.e., the elliptic case), they are measurable in $x^{1}$ and VMO in $x^{\prime} \in \mathbb{R}^{d-1}$. To state this assumption precisely, we introduce the following notations. Let

$$
\begin{gathered}
B_{r}(x)=\left\{y \in \mathbb{R}^{d}:|x-y|<r\right\}, \quad B_{r}^{\prime}\left(x^{\prime}\right)=\left\{y^{\prime} \in \mathbb{R}^{d-1}:\left|x^{\prime}-y^{\prime}\right|<r\right\}, \\
\Gamma_{r}(x)=\left(x^{1}-r, x^{1}+r\right) \times B_{r}^{\prime}\left(x^{\prime}\right), \\
Q_{r}(t, x)=\left(t, t+r^{2}\right) \times B_{r}(x), \quad \Lambda_{r}(t, x)=\left(t, t+r^{2}\right) \times \Gamma_{r}(x) .
\end{gathered}
$$

Set $B_{r}=B_{r}(0), B_{r}^{\prime}=B_{r}^{\prime}(0), Q_{r}=Q_{r}(0)$ and so on. By $\left|B_{r}^{\prime}\right|$ we mean the $d-1$ dimensional volume of $B_{r}^{\prime}(0)$. Denote

$$
\begin{gathered}
\operatorname{osc}_{x^{\prime}}\left(a^{i j}, \Lambda_{r}(t, x)\right)=r^{-3}\left|B_{r}^{\prime}\right|^{-2} \int_{t}^{t+r^{2}} \int_{x^{1}-r}^{x^{1}+r} A_{x^{\prime}}^{i j}(s, \tau) d \tau d s, \\
\operatorname{osc}_{\left(t, x^{\prime}\right)}\left(a^{i j}, \Lambda_{r}(t, x)\right)=r^{-5}\left|B_{r}^{\prime}\right|^{-2} \int_{x^{1}-r}^{x^{1}+r} A_{\left(t, x^{\prime}\right)}^{i j}(\tau) d \tau,
\end{gathered}
$$


where

$$
\begin{gathered}
A_{x^{\prime}}^{i j}(s, \tau)=\int_{y^{\prime}, z^{\prime} \in B_{r}^{\prime}\left(x^{\prime}\right)}\left|a^{i j}\left(s, \tau, y^{\prime}\right)-a^{i j}\left(s, \tau, z^{\prime}\right)\right| d y^{\prime} d z^{\prime}, \\
A_{\left(t, x^{\prime}\right)}^{i j}(\tau)=\int_{\sigma, \varrho \in\left(t, t+r^{2}\right)} \int_{y^{\prime}, z^{\prime} \in B_{r}^{\prime}\left(t, x^{\prime}\right)}\left|a^{i j}\left(\sigma, \tau, y^{\prime}\right)-a^{i j}\left(\varrho, \tau, z^{\prime}\right)\right| d y^{\prime} d z^{\prime} d \sigma d \varrho .
\end{gathered}
$$

Also denote

$$
\begin{gathered}
\mathcal{O}_{R}^{x^{\prime}}\left(a^{i j}\right)=\sup _{(t, x) \in \mathbb{R}^{d+1}} \sup _{r \leq R} \operatorname{osc}_{x^{\prime}}\left(a^{i j}, \Lambda_{r}(t, x)\right), \\
\mathcal{O}_{R}^{\left(t, x^{\prime}\right)}\left(a^{i j}\right)=\sup _{(t, x) \in \mathbb{R}^{d+1}} \sup _{r \leq R} \operatorname{osc}_{\left(t, x^{\prime}\right)}\left(a^{i j}, \Lambda_{r}(t, x)\right) .
\end{gathered}
$$

Finally set

$$
a_{R}^{\#}=\mathcal{O}_{R}^{\left(t, x^{\prime}\right)}\left(a^{11}\right)+\sum_{i \neq 1 \text { or } j \neq 1} \mathcal{O}_{R}^{x^{\prime}}\left(a^{i j}\right) .
$$

If elliptic equations are considered, we set

$$
\operatorname{osc}_{x^{\prime}}\left(a^{i j}, \Gamma_{r}(x)\right)=r^{-1}\left|B_{r}^{\prime}\right|^{-2} \int_{x^{1}-r}^{x^{1}+r} \int_{y^{\prime}, z^{\prime} \in B_{r}^{\prime}\left(x^{\prime}\right)}\left|a^{i j}\left(\tau, y^{\prime}\right)-a^{i j}\left(\tau, z^{\prime}\right)\right| d y^{\prime} d z^{\prime} d \tau
$$

and

$$
a_{R}^{\#}=\sup _{x \in \mathbb{R}^{d}} \sup _{r \leq R} \operatorname{osc}_{x^{\prime}}\left(a^{i j}, \Gamma_{r}(x)\right)
$$

Assumption 2.2. There is a continuous function $\omega(r)$ defined on $[0, \infty)$ such that $\omega(0)=0$ and $a_{R}^{\#} \leq \omega(R)$ for all $R \in[0, \infty)$.

By $W_{q, p}^{1,2}(\mathcal{D})$, where $\mathcal{D}$ is an open set in $\mathbb{R}^{d+1}$, we mean the collection of all functions defined on $\mathcal{D}$ such that

$$
\|u\|_{W_{q, p}^{1,2}(\mathcal{D})}:=\|u\|_{L_{q, p}(\mathcal{D})}+\left\|u_{x}\right\|_{L_{q, p}(\mathcal{D})}+\left\|u_{x x}\right\|_{L_{q, p}(\mathcal{D})}+\left\|u_{t}\right\|_{L_{q, p}(\mathcal{D})}<\infty,
$$

where $L_{q, p}(\mathcal{D})$ is defined in (4). For elliptic equations, we say $u \in W_{q, p}^{2}(\Omega)$ if $u, u_{x}$, $u_{x x} \in L_{q, p}(\Omega)$, where $\Omega$ is an open set in $\mathbb{R}^{d}$. Again note that $W_{p, p}^{1,2}(\mathcal{D})=W_{p}^{1,2}(\mathcal{D})$ and $W_{p, p}^{2}(\Omega)=W_{p}^{2}(\Omega)$. We denote the parabolic and elliptic differential operators by $L$ and $E$, respectively, that is,

$$
\begin{gathered}
L u=u_{t}+a^{i j} u_{x^{i} x^{j}}+b^{i} u_{x^{i}}+c u, \\
E u=a^{i j} u_{x^{i} x^{j}}+b^{i} u_{x^{i}}+c u .
\end{gathered}
$$

The following are our main results. First we state our result about parabolic equations in the whole space.

THEOREM 2.3. Let $q>p \geq 2,0<T<\infty$, and the coefficients of $L$ satisfy Assumption 2.1 and 2.2. In addition, we assume that, in case $p=2$, $a^{i j}$ are independent of $x^{\prime} \in \mathbb{R}^{d-1}$. Then for any $f \in L_{q, p}\left((0, T) \times \mathbb{R}^{d}\right)$, there exists a unique 
$u \in W_{q, p}^{1,2}\left((0, T) \times \mathbb{R}^{d}\right)$ such that $u(T, x)=0$ and $L u=f$ in $(0, T) \times \mathbb{R}^{d}$. Furthermore, there is a constant $N$, depending only on $d_{1}, d_{2}, \delta, K, p, q, T$, and the function $\omega$, such that

$$
\|u\|_{W_{q, p}^{1,2}\left((0, T) \times \mathbb{R}^{d}\right)} \leq N\|L u\|_{L_{q, p}\left((0, T) \times \mathbb{R}^{d}\right)}
$$

for any $u \in W_{q, p}^{1,2}\left((0, T) \times \mathbb{R}^{d}\right)$ satisfying $u(T, x)=0$.

We have the following result for elliptic equations in the whole space.

TheOREm 2.4. Let $q>p \geq 2$ and the coefficients of E satisfy Assumption 2.1 and 2.2. In addition, we assume that, in case $p=2$, $a^{i j}$ are independent of $x^{\prime} \in \mathbb{R}^{d-1}$. Then there exist constants $\lambda_{0} \geq 0$ and $N$, depending only on $d_{1}, d_{2}, \delta, K, p, q$, and the function $\omega$, such that

$$
\lambda\|u\|_{L_{q, p}\left(\mathbb{R}^{d}\right)}+\sqrt{\lambda}\left\|u_{x}\right\|_{L_{q, p}\left(\mathbb{R}^{d}\right)}+\left\|u_{x x}\right\|_{L_{q, p}\left(\mathbb{R}^{d}\right)} \leq N\|E u-\lambda u\|_{L_{q, p}\left(\mathbb{R}^{d}\right)}
$$

for any $u \in W_{q, p}^{2}\left(\mathbb{R}^{d}\right)$ and $\lambda \geq \lambda_{0}$. Moreover, for any $\lambda>\lambda_{0}$ and $f \in L_{q, p}\left(\mathbb{R}^{d}\right)$, there exists a unique $u \in W_{q, p}^{2}\left(\mathbb{R}^{d}\right)$ such that $E u-\lambda u=f$ in $\mathbb{R}^{d}$.

We present results about equations on a half space. Recall the definition of $\mathbb{R}_{+}^{d}$ given in the introduction. The proofs of the following two theorems show how useful is the assumption that $a^{i j}$ are allowed to be only measurable in one spatial direction. Since their proofs are almost the same, we here give only a proof of Theorem 2.5. Also see Theorem 2.7 in [9] and Theorem 2.7 in [8].

TheOREM 2.5. Let $q>p \geq 2,0<T<\infty$, and the coefficients of $L$ satisfy Assumption 2.1 and 2.2. In addition, we assume that, in case $p=2, a^{i j}$ are independent of $x^{\prime} \in \mathbb{R}^{d-1}$. Then for any $f \in L_{q, p}\left((0, T) \times \mathbb{R}_{+}^{d}\right)$, there exists a unique $u \in W_{q, p}^{1,2}\left((0, T) \times \mathbb{R}_{+}^{d}\right)$ such that $u(T, x)=u\left(t, 0, x^{\prime}\right)=0$ and $L u=f$ in $(0, T) \times \mathbb{R}_{+}^{d}$.

Proof. Define a new operator $\hat{L}$ by

$$
\hat{L}=\frac{\partial}{\partial t}+\hat{a}^{i j} \frac{\partial^{2}}{\partial x^{i} \partial x^{j}}+\hat{b}^{i} \frac{\partial}{\partial x^{i}}+\hat{c},
$$

where $\hat{a}^{i j}, \hat{b}^{i}$, and $\hat{c}$ are either even or odd extensions of $a^{i j}, b^{i}$, and $c$. Specifically, for $i=j=1$ or $i, j \in\{2, \cdots, d\}$, the coefficients $\hat{a}^{i j}$ are even extensions of $a^{i j}$ :

$$
\hat{a}^{i j}\left(t, x^{1}, x^{\prime}\right)=a^{i j}\left(t, x^{1}, x^{\prime}\right) I_{x^{1} \geq 0}+a^{i j}\left(t,-x^{1}, x^{\prime}\right) I_{x^{1}<0} .
$$

For $j=2, \cdots, d$, the coefficients $\hat{a}^{1 j}$ are odd extensions of $a^{1 j}$ :

$$
\hat{a}^{1 j}\left(t, x^{1}, x^{\prime}\right)=a^{1 j}\left(t, x^{1}, x^{\prime}\right) I_{x^{1} \geq 0}-a^{1 j}\left(t,-x^{1}, x^{\prime}\right) I_{x^{1}<0} .
$$

Similarly, $\hat{b}^{1}$ is the odd extension of $b^{1}$, and $\hat{b}^{i}, j=2, \cdots d$, as well as $\hat{c}$ are the even extensions of $b^{j}$ and $c$. We see that the coefficients $\hat{a}^{i j}, \hat{b}^{i}$, and $\hat{c}$ satisfy Assumption 2.1. In addition, the coefficients $\hat{a}^{i j}$ satisfy Assumption 2.2 with $2 \omega$. Let $\hat{f}$ be the odd extension of $f$. By Theorem 2.3 there exists a unique $u \in W_{q, p}^{1,2}\left((0, T) \times \mathbb{R}^{d}\right)$ such that $\hat{L} u=\hat{f}$ and $u(T, x)=0$. It is easy to check that $-u\left(t,-x^{1}, x^{\prime}\right) \in W_{q, p}^{1,2}\left((0, T) \times \mathbb{R}^{d}\right)$ also satisfies the same equation, so by uniqueness we have $u\left(t, x^{1}, x^{\prime}\right)=-u\left(t,-x^{1}, x^{\prime}\right)$. From this it follows that $u\left(t, 0, x^{\prime}\right)=0$ for $\left(t, x^{\prime}\right) \in(0, T) \times \mathbb{R}^{d-1}$. This together with the fact that $u(T, 0)=0$ shows that that $u$, as a function defined on $(0, T) \times R_{+}^{d}$, 
is a solution to $L u=f$ satisfying $u(T, x)=u(t, 0, x)=0$. Uniqueness follows from the fact that the odd extension of a solution belongs to $W_{q, p}^{1,2}\left((0, T) \times \mathbb{R}^{d}\right)$ and the uniqueness of solutions to equations on $(0, T) \times \mathbb{R}^{d}$. $\square$

TheOREm 2.6. Let $q>p \geq 2$ and the coefficients of $E$ satisfy Assumption 2.1 and 2.2. In addition, we assume that, in case $p=2$, $a^{i j}$ are independent of $x^{\prime} \in \mathbb{R}^{d-1}$. Then there exist constants $\lambda_{0} \geq 0$ and $N$, depending only on $d_{1}, d_{2}, \delta, K, p, q$, and the function $\omega$, such that, for any $\lambda>\lambda_{0}$ and $f \in L_{q, p}\left(\mathbb{R}_{+}^{d}\right)$, there exists a unique $u \in W_{q, p}^{2}\left(\mathbb{R}_{+}^{d}\right)$ satisfying $u\left(0, x^{\prime}\right)=0$ and $E u-\lambda u=f$ in $\mathbb{R}_{+}^{d}$.

REMARK 2.7. The above four theorems for the case $q=p \geq 2$ follow from the results in $[8,9,7]$. If $q=p=2$, all of the coefficients $a^{i j}(t, x)$ for the parabolic operator $L$ are further allowed to be measurable in $\left(t, x^{1}\right)$ (see [9]) as long as they are functions of only $\left(t, x^{1}\right)$. In the above theorems, the condition that $a^{i j}$ are independent of $x^{\prime} \in \mathbb{R}^{d-1}$ can be obviously replaced by the uniform continuity of $a^{i j}$ as functions of $x^{\prime} \in \mathbb{R}^{d-1}$ uniformly in $\left(t, x^{1}\right)$. Regarding Theorem 2.5 and 2.6, appropriate $L_{q, p^{-}}$ estimates can be stated. Moreover, it is possible to replace the Dirichlet boundary condition by the Neumann boundary condition. That is, in Theorem 2.5 the condition $u(t, 0, x)=0$ can be replaced by $u_{x^{1}}(t, 0, x)=0$. In Theorem $2.6 u_{x^{1}}(0, x)=0$ in place of $u(0, x)=0$. The proofs are the same, but instead of the odd extension of $f$, one has to use its even extension. For details, see Theorem 2.8 in [8] or Theorem 2.8 in [9].

3. Parabolic equations with $a^{i j}$ measurable in $x^{1} \in \mathbb{R}$ and VMO in $\left(t, x^{\prime}\right) \in \mathbb{R}^{d}$. To move toward the proofs of the main results above, we first need to deal with the parabolic operator $L$ when the coefficients $a^{i j}\left(t, x^{1}, x^{\prime}\right)$ are measurable in $x^{1} \in \mathbb{R}$ and VMO in $\left(t, x^{\prime}\right) \in \mathbb{R}^{d}$. Note that in Theorem 2.3 and 2.5 all coefficients $a^{i j}\left(t, x^{1}, x^{\prime}\right)$ except $a^{11}$ are assumed to be measurable in $\left(t, x^{1}\right)$ and VMO in $x^{\prime}$, so the class of coefficients we consider in this section is less general than those in Theorem 2.3 and 2.5. To state this assumption on the coefficients $a^{i j}$ precisely, set

$$
a_{R}^{\#\left(t, x^{\prime}\right)}=\sum_{i, j=1} \mathcal{O}_{R}^{\left(t, x^{\prime}\right)}\left(a^{i j}\right)
$$

Assumption 3.1. There is a continuous function $\omega(r)$ defined on $[0, \infty)$ such that $\omega(0)=0$ and $a_{R}^{\#\left(t, x^{\prime}\right)} \leq \omega(R)$ for all $R \in[0, \infty)$.

The following is the main result of this section.

TheOREM 3.2. Let $q>p \geq 2,0<T<\infty$, and the coefficients of $L$ satisfy Assumption 2.1 and 3.1. In addition, we assume that, in case $p=2$, $a^{i j}$ are independent of $x^{\prime} \in \mathbb{R}^{d-1}$. Then for any $f \in L_{q, p}\left((0, T) \times \mathbb{R}^{d}\right)$, there exists a unique $u \in W_{q, p}^{1,2}\left((0, T) \times \mathbb{R}^{d}\right)$ such that $u(T, x)=0$ and $L u=f$ in $(0, T) \times \mathbb{R}^{d}$. Furthermore, there is a constant $N$, depending only on $d_{1}, d_{2}, \delta, K, p, q, T$, and the function $\omega$, such that

$$
\|u\|_{W_{q, p}^{1,2}\left((0, T) \times \mathbb{R}^{d}\right)} \leq N\|L u\|_{L_{q, p}\left((0, T) \times \mathbb{R}^{d}\right)}
$$

for any $u \in W_{q, p}^{1,2}\left((0, T) \times \mathbb{R}^{d}\right)$ satisfying $u(T, x)=0$.

This theorem is proved in the next section after presenting some preliminary results. Throughout this section, we set

$$
\mathcal{L}_{\lambda} u=u_{t}+a^{i j}\left(x^{1}\right) u_{x^{i} x^{j}}-\lambda u,
$$


where $\lambda \geq 0$ and $a^{i j}$ are measurable functions of only $x^{1} \in \mathbb{R}$ satisfying Assumption 2.1. We start with a theorem which can be derived from results in [9].

Theorem 3.3. Let $p \geq 2$ and $T \in[-\infty, \infty)$. For any $\lambda>0$ and $f \in L_{p}((T, \infty) \times$ $\left.\mathbb{R}^{d}\right)$, there exists a unique solution $u \in W_{p}^{1,2}\left((T, \infty) \times \mathbb{R}^{d}\right)$ to the equation $\mathcal{L}_{\lambda} u=f$. Furthermore, there is a constant $N=N(d, p, \delta)$ such that, for any $\lambda \geq 0$ and $u \in$ $W_{p}^{1,2}\left((T, \infty) \times \mathbb{R}^{d}\right)$, we have

$$
\begin{gathered}
\left\|u_{t}\right\|_{L_{p}\left((T, \infty) \times \mathbb{R}^{d}\right)}+\left\|u_{x x}\right\|_{L_{p}\left((T, \infty) \times \mathbb{R}^{d}\right)}+\sqrt{\lambda}\left\|u_{x}\right\|_{L_{p}\left((T, \infty) \times \mathbb{R}^{d}\right)} \\
+\lambda\|u\|_{L_{p}\left((T, \infty) \times \mathbb{R}^{d}\right)} \leq N\left\|\mathcal{L}_{\lambda} u\right\|_{L_{p}\left((T, \infty) \times \mathbb{R}^{d}\right)} .
\end{gathered}
$$

If $T=-\infty$, this theorem is obtained from Theorem 3.2 in [9] for $p=2$ and Lemma $5.3^{*}$ in [9] for $p>2$. For the case $T \in(-\infty, \infty)$, we use the case $T=-\infty$ and the argument following Corollary 5.14 in [14].

The following three lemmas are $L_{p}$-versions of Lemma $4.2,4.3$, and 4.4 in [9]. Since the estimate in Theorem 3.3 is available, their proofs can be done by repeating the proofs of Lemma 4.2, 4.3, and 4.4 in [9] with $p$ in place of 2 .

Lemma 3.4. Let $p \in[2, \infty)$. For any $u \in W_{p, l o c}^{1,2}\left(\mathbb{R}^{d+1}\right)$, we have

$$
\left\|u_{t}\right\|_{L_{p}\left(Q_{r}\right)}+\left\|u_{x x}\right\|_{L_{p}\left(Q_{r}\right)}+\left\|u_{x}\right\|_{L_{p}\left(Q_{r}\right)} \leq N\left(\left\|\mathcal{L}_{0} u\right\|_{L_{p}\left(Q_{R}\right)}+\|u\|_{L_{p}\left(Q_{R}\right)}\right),
$$

where $0<r<R<\infty$ and $N=N(d, p, \delta, r, R)$.

Lemma 3.5. Let $p \in[2, \infty), 0<r<R<\infty$, and $\gamma=\left(\gamma^{1}, \cdots, \gamma^{d}\right)$ be a multiindex such that $\gamma^{1}=0,1,2$. If $v \in C_{l o c}^{\infty}\left(\mathbb{R}^{d+1}\right)$ is a function such that $\mathcal{L}_{0} v=0$ in $Q_{R}$, then

$$
\int_{Q_{r}}\left|D_{t}^{m} D_{x}^{\gamma} v\right|^{p} d x d t \leq N \int_{Q_{R}}|v|^{p} d x d t
$$

where $m$ is a nonnegative integer and $N=N(d, p, \delta, \gamma, m, r, R)$.

Lemma 3.6. Let $p \geq 2$ and $v \in C_{\text {loc }}^{\infty}\left(\mathbb{R}^{d+1}\right)$ be a function such that $\mathcal{L}_{0} v=0$ in $Q_{4}$. Then

$$
\sup _{Q_{1}}\left|v_{t t}\right|+\sup _{Q_{1}}\left|v_{t x}\right|+\sup _{Q_{1}}\left|v_{t x x^{\prime}}\right|+\sup _{Q_{1}}\left|v_{x x x^{\prime}}\right| \leq N\|v\|_{L_{p}\left(Q_{4}\right)},
$$

where $N=N(d, p, \delta)$.

The proofs of the lemmas and theorem below follow the ideas in [14], specifically, those in the proofs of Lemma 5.9, Theorem 5.10, and Theorem 5.1 in [14].

Lemma 3.7. Let $p \geq 2$ and $\lambda \geq 0$. For every $v \in C_{\text {loc }}^{\infty}\left(\mathbb{R}^{d+1}\right)$ such that $\mathcal{L}_{\lambda} v=0$ in $Q_{4}$, we have

$$
\begin{aligned}
& \sup _{Q_{1}}\left|v_{t t}\right|+\sup _{Q_{1}}\left|v_{t x}\right|+\sup _{Q_{1}}\left|v_{t x x^{\prime}}\right|+\sup _{Q_{1}}\left|v_{x x x^{\prime}}\right| \\
\leq & N(d, p, \delta)\left(\left\|v_{x x}\right\|_{L_{p}\left(Q_{4}\right)}+\left\|v_{t}\right\|_{L_{p}\left(Q_{4}\right)}+\sqrt{\lambda}\left\|v_{x}\right\|_{L_{p}\left(Q_{4}\right)}\right) .
\end{aligned}
$$

\footnotetext{
*In fact, Lemma 5.3 in [9] says that the estimate in Theorem 3.3 holds for all $\lambda \geq \lambda_{0} \geq 0$, where $\lambda_{0}$ is not necessarily 0 . However, since the coefficients $a^{i j}$ of $\mathcal{L}_{\lambda}$ are measurable functions of only $x^{1} \in \mathbb{R}$ and $b^{i}=c=0$, it can be proved, using a dilation argument, that $\lambda_{0}=0$ in our case.
} 
Proof. We first note that, in case $\lambda=0$, by Lemma 3.6

$$
I:=\sup _{Q_{1}}\left|v_{t t}\right|+\sup _{Q_{1}}\left|v_{t x}\right|+\sup _{Q_{1}}\left|v_{t x x^{\prime}}\right|+\sup _{Q_{1}}\left|v_{x x x^{\prime}}\right| \leq N\|v\|_{L_{p}\left(Q_{4}\right)},
$$

The function $u:=v-(v)_{Q_{4}}-x^{i}\left(v_{x^{i}}\right)_{Q_{4}}$ can replace $v$ in the above inequality since $\mathcal{L}_{0} u=0$ in $Q_{4}$. This together with the fact that $D_{t}^{m} D_{x}^{\gamma} v=D_{t}^{m} D_{x}^{\gamma} u$ for $m \geq 1$ or $|\gamma| \geq 2$ gives

$$
I \leq N\left\|v-(v)_{Q_{4}}-x^{i}\left(v_{x^{i}}\right)_{Q_{4}}\right\|_{L_{p}\left(Q_{4}\right)} .
$$

This and Lemma 5.4 in [14] prove the inequality in the lemma for $\lambda=0$.

In case $\lambda>0$, we extend $v(t, x)$ to a function defined on $\mathbb{R}^{d+2}$. Specifically, set

$$
\mathbf{v}(t, x, \xi)=v(t, x) \cos (\sqrt{\lambda} \xi),
$$

where $\xi \in \mathbb{R}$ and $(t, x, \xi) \in \mathbb{R}^{d+2}$. Notice that

$$
\begin{gathered}
\mathcal{L}_{0} \mathbf{v}+\mathbf{v}_{\xi \xi}=0 \quad \text { in } \mathbf{Q}_{4}, \\
D_{t}^{m} D_{x}^{\gamma} v(t, x)=D_{t}^{m} D_{x}^{\gamma} \mathbf{v}(t, x, 0), \quad \sup _{Q_{1}}\left|D_{t}^{m} D_{x}^{\gamma} v\right| \leq \sup _{\mathbf{Q}_{1}}\left|D_{t}^{m} D_{x}^{\gamma} \mathbf{v}\right|,
\end{gathered}
$$

where $\mathbf{Q}_{r}=\left(0, r^{2}\right) \times\left\{(x, \xi) \in \mathbb{R}^{d+1}:|x|^{2}+\xi^{2}<r^{2}\right\}$. Thus by the argument above for the case $\lambda=0$ we have

$$
I \leq N\left(\left\|\mathbf{v}_{x x}\right\|_{L_{p}\left(\mathbf{Q}_{4}\right)}+\left\|\mathbf{v}_{x \xi}\right\|_{L_{p}\left(\mathbf{Q}_{4}\right)}+\left\|\mathbf{v}_{\xi \xi}\right\|_{L_{p}\left(\mathbf{Q}_{4}\right)}+\left\|\mathbf{v}_{t}\right\|_{L_{p}\left(\mathbf{Q}_{4}\right)}\right) .
$$

Note that, for example,

$$
\mathbf{v}_{x x}=v_{x x} \cos (\sqrt{\lambda} \xi), \quad \mathbf{v}_{x \xi}=-\sqrt{\lambda} v_{x} \sin (\sqrt{\lambda} \xi), \quad \mathbf{v}_{\xi \xi}=-\lambda v \cos (\sqrt{\lambda} \xi) .
$$

Therefore, the right-hand side of the inequality (6) is not greater than a constant times

$$
\left\|v_{x x}\right\|_{L_{p}\left(Q_{4}\right)}+\left\|v_{t}\right\|_{L_{p}\left(Q_{4}\right)}+\sqrt{\lambda}\left\|v_{x}\right\|_{L_{p}\left(Q_{4}\right)}+\lambda\|v\|_{L_{p}\left(Q_{4}\right)} .
$$

This is bounded by the right-hand side of the inequality in the lemma (note that $\lambda v=\mathcal{L}_{0} v$ in $\left.Q_{4}\right)$. The lemma is proved. $\mathrm{Q}$

Lemma 3.8. Let $p \geq 2, \lambda \geq 0, \kappa \geq 4$, and $r \in(0, \infty)$. Let $v \in C_{\text {loc }}^{\infty}\left(\mathbb{R}^{d+1}\right)$ be such that $\mathcal{L}_{\lambda} v=0$ in $Q_{\kappa r}$. Then there is a constant $N$, depending only on $d, p$, and $\delta$, such that

$$
\begin{aligned}
f_{Q_{r}}\left|v_{t}(t, x)-\left(v_{t}\right)_{Q_{r}}\right|^{p} d x d t+f_{Q_{r}} & \left|v_{x x^{\prime}}(t, x)-\left(v_{x x^{\prime}}\right)_{Q_{r}}\right|^{p} d x d t \\
& \leq N \kappa^{-p}\left(\left|v_{x x}\right|^{p}+\left|v_{t}\right|^{p}+\lambda^{p / 2}\left|v_{x}\right|^{p}\right)_{Q_{\kappa r}} .
\end{aligned}
$$

Proof. Due to a dilation argument (see the proof of Theorem 5.10 in [14]), it is enough to prove the inequality (7) when $r=1$.

For $v \in C_{\text {loc }}^{\infty}\left(\mathbb{R}^{d+1}\right)$ such that $\mathcal{L}_{\lambda} v=0$ in $Q_{\kappa}, \kappa \geq 4$, set

$$
\check{v}(t, x)=v\left(\left(\frac{\kappa}{4}\right)^{2} t, \frac{\kappa}{4} x\right), \quad \check{a}^{i j}\left(x^{1}\right)=a^{i j}\left(\kappa x^{1} / 4\right) .
$$


Then

$$
\begin{gathered}
\check{\mathcal{L}}_{\left(\frac{\kappa}{4}\right)^{2} \lambda} \check{v}(t, x):=\left(\frac{\partial}{\partial t}+\check{a}^{i j}\left(x^{1}\right) \frac{\partial^{2}}{\partial x^{i} \partial x^{j}}-\left(\frac{\kappa}{4}\right)^{2} \lambda\right) \check{v}(t, x) \\
=\left(\frac{\kappa}{4}\right)^{2}\left(\mathcal{L}_{\lambda} v\right)\left(\left(\frac{\kappa}{4}\right)^{2} t, \frac{\kappa}{4} x\right)=0 \quad \text { in } \quad Q_{4} .
\end{gathered}
$$

Thus by Lemma 3.7, it follows that

$$
\check{I} \leq N\left(\left\|\check{v}_{x x}\right\|_{L_{p}\left(Q_{4}\right)}+\left\|\check{v}_{t}\right\|_{L_{p}\left(Q_{4}\right)}+\frac{\kappa}{4} \sqrt{\lambda}\left\|\check{v}_{x}\right\|_{L_{p}\left(Q_{4}\right)}\right),
$$

where

$$
\check{I}:=\sup _{Q_{1}}\left|\check{v}_{t t}\right|+\sup _{Q_{1}}\left|\check{v}_{t x}\right|+\sup _{Q_{1}}\left|\check{v}_{t x x^{\prime}}\right|+\sup _{Q_{1}}\left|\check{v}_{x x x^{\prime}}\right|
$$

Note that

$$
(4 / \kappa)^{3} \check{I}=(\kappa / 4)\left(\sup _{Q_{\kappa / 4}}\left|v_{t t}\right|+\sup _{Q_{\kappa / 4}}\left|v_{t x x^{\prime}}\right|\right)+\sup _{Q_{\kappa / 4}}\left|v_{t x}\right|+\sup _{Q_{\kappa / 4}}\left|v_{x x x^{\prime}}\right| .
$$

Using this, the inequality (8), and the fact that $\kappa \geq 4$, we have

$$
\begin{gathered}
f_{Q_{1}}\left|v_{t}(t, x)-\left(v_{t}\right)_{Q_{1}}\right|^{p} d x d t+f_{Q_{1}}\left|v_{x x^{\prime}}(t, x)-\left(v_{x x^{\prime}}\right)_{Q_{1}}\right|^{p} d x d t \\
\leq N\left(\sup _{Q_{\kappa / 4}}\left|v_{t t}\right|+\sup _{Q_{\kappa / 4}}\left|v_{t x}\right|+\sup _{Q_{\kappa / 4}}\left|v_{t x x^{\prime}}\right|+\sup _{Q_{\kappa / 4}}\left|v_{x x x^{\prime}}\right|\right)^{p} \leq N \kappa^{-3 p} \check{I}^{p} \\
\leq N \kappa^{-3 p}\left(\left\|\check{v}_{x x}\right\|_{L_{p}\left(Q_{4}\right)}^{p}+\left\|\check{v}_{t}\right\|_{L_{p}\left(Q_{4}\right)}^{p}+\kappa^{p} \lambda^{p / 2}\left\|\check{v}_{x}\right\|_{L_{p}\left(Q_{4}\right)}^{p}\right) \\
=N \kappa^{-p}\left(\left|v_{x x}\right|^{p}+\left|v_{t}\right|^{p}+\lambda^{p / 2}\left|v_{x}\right|^{p}\right)_{L_{p}\left(Q_{\kappa}\right)} .
\end{gathered}
$$

This finishes the proof.

Theorem 3.9. Let $p \geq 2$. Then there is a constant $N$, depending only on $d, p$, and $\delta$, such that, for any $u \in W_{p}^{1,2}\left(\mathbb{R}^{d+1}\right), r \in(0, \infty)$, and $\kappa \geq 8$,

$$
\begin{gathered}
f_{Q_{r}}\left|u_{t}(t, x)-\left(u_{t}\right)_{Q_{r}}\right|^{p} d x d t+f_{Q_{r}}\left|u_{x x^{\prime}}(t, x)-\left(u_{x x^{\prime}}\right)_{Q_{r}}\right|^{p} d x d t \\
\leq N \kappa^{d+2}\left(\left|\mathcal{L}_{0} u\right|^{p}\right)_{Q_{\kappa r}}+N \kappa^{-p}\left(\left|u_{x x}\right|^{p}\right)_{Q_{\kappa r}} .
\end{gathered}
$$

Proof. Since $C_{0}^{\infty}\left(\mathbb{R}^{d+1}\right)$ is dense in $W_{p}^{1,2}\left(\mathbb{R}^{d+1}\right)$, it is enough to have $u \in$ $C_{0}^{\infty}\left(\mathbb{R}^{d+1}\right)$. In addition, we can assume that $a^{i j}\left(x^{1}\right)$ are infinitely differentiable. Take a $\lambda>0$ and, for $u \in C_{0}^{\infty}\left(\mathbb{R}^{d+1}\right)$, let

$$
f:=f_{\lambda}=\mathcal{L}_{\lambda} u
$$


We see $f \in C_{0}^{\infty}\left(\mathbb{R}^{d+1}\right)$. For given $r>0$ and $\kappa \geq 8$, let $\eta \in C_{0}^{\infty}\left(\mathbb{R}^{d+1}\right)$ be a function such that $\eta=1$ on $Q_{\kappa r / 2}$ and $\eta=0$ outside $\left(-(\kappa r)^{2},(\kappa r)^{2}\right) \times B_{\kappa r}$. Also let

$$
g:=f \eta, \quad h:=f(1-\eta) .
$$

Then by Theorem 3.3 there exists a unique solution $v \in W_{p}^{1,2}\left(\mathbb{R}^{d+1}\right.$ ) (note that $\lambda>0$ ) to the equation $\mathcal{L}_{\lambda} v=h$. From the classical theory we see that the function $v$ is infinitely differentiable. Moreover, since $\mathcal{L}_{\lambda} v=h=0$ in $Q_{\kappa r / 2}$ and $\kappa / 2 \geq 4$, by Lemma 3.8, we have

$$
\begin{gathered}
f_{Q_{r}}\left|v_{t}(t, x)-\left(v_{t}\right)_{Q_{r}}\right|^{p} d x d t+f_{Q_{r}}\left|v_{x x^{\prime}}(t, x)-\left(v_{x x^{\prime}}\right)_{Q_{r}}\right|^{p} d x d t \\
\leq N \kappa^{-p}\left(\left|v_{x x}\right|^{p}+\left|v_{t}\right|^{p}+\lambda^{p / 2}\left|v_{x}\right|^{p}\right)_{Q_{\kappa r / 2}} \leq N \kappa^{-p}\left(\left|v_{x x}\right|^{p}+\left|v_{t}\right|^{p}+\lambda^{p / 2}\left|v_{x}\right|^{p}\right)_{Q_{\kappa r}} .
\end{gathered}
$$

Set $w:=u-v \in W_{p}^{1,2}\left(\mathbb{R}^{d+1}\right)$. Then from the above inequality it follows that

$$
\begin{gathered}
f_{Q_{r}}\left|u_{x x^{\prime}}(t, x)-\left(u_{x x^{\prime}}\right)_{Q_{r}}\right|^{p} d x d t \\
\leq 2^{p} f_{Q_{r}}\left|w_{x x^{\prime}}(t, x)-\left(w_{x x^{\prime}}\right)_{Q_{r}}\right|^{p} d x d t+2^{p} f_{Q_{r}}\left|v_{x x^{\prime}}(t, x)-\left(v_{x x^{\prime}}\right)_{Q_{r}}\right|^{p} d x d t \\
\leq N\left(\left|w_{x x^{\prime}}\right|^{p}\right)_{Q_{r}}+N \kappa^{-p}\left(\left|v_{x x}\right|^{p}+\left|v_{t}\right|^{p}+\lambda^{p / 2}\left|v_{x}\right|^{p}\right)_{Q_{\kappa r}} .
\end{gathered}
$$

Similar inequalities are possible with $u_{t}$ in place of $u_{x x^{\prime}}$. Thus we have

$$
\begin{aligned}
f_{Q_{r}} \mid u_{t}(t, x)- & \left.\left(u_{t}\right)_{Q_{r}}\right|^{p} d x d t+f_{Q_{r}}\left|u_{x x^{\prime}}(t, x)-\left(u_{x x^{\prime}}\right)_{Q_{r}}\right|^{p} d x d t \\
& \leq N\left(\left|w_{t}\right|^{p}+\left|w_{x x}\right|^{p}\right)_{Q_{r}}+N \kappa^{-p}\left(\left|v_{x x}\right|^{p}+\left|v_{t}\right|^{p}+\lambda^{p / 2}\left|v_{x}\right|^{p}\right)_{Q_{\kappa r}} .
\end{aligned}
$$

Now we observe that

$$
\mathcal{L}_{\lambda} w=\mathcal{L}_{\lambda}(u-v)=f-h=g
$$

and, by Theorem 3.3,

$$
\begin{gathered}
\int_{Q_{r}}\left|w_{t}\right|^{p} d x d t+\int_{Q_{r}}\left|w_{x x}\right|^{p} d x d t \\
\leq\left\|w_{t}\right\|_{L_{p}\left((0, \infty) \times \mathbb{R}^{d}\right)}^{p}+\left\|w_{x x}\right\|_{L_{p}\left((0, \infty) \times \mathbb{R}^{d}\right)}^{p}+\lambda^{p / 2}\left\|w_{x}\right\|_{L_{p}\left((0, \infty) \times \mathbb{R}^{d}\right)}^{p} \\
\leq N\|g\|_{L_{p}\left((0, \infty) \times \mathbb{R}^{d}\right)}^{p}=N \int_{Q_{\kappa r}}|g|^{p} d x d t \leq N \int_{Q_{\kappa r}}|f|^{p} d x d t .
\end{gathered}
$$

From this we see that

$$
\left(\left|w_{t}\right|^{p}\right)_{Q_{r}}+\left(\left|w_{x x}\right|^{p}\right)_{Q_{r}} \leq N \kappa^{d+2}\left(|f|^{p}\right)_{Q_{\kappa r}},
$$




$$
\left(\left|w_{x x}\right|^{p}+\left|w_{t}\right|^{p}+\lambda^{p / 2}\left|w_{x}\right|^{p}\right)_{Q_{\kappa r}} \leq N\left(|f|^{p}\right)_{Q_{\kappa r}} .
$$

Now we use the these inequalities as well as the inequality (9). We also use the fact $u=w+v$ and $\kappa \geq 8$. Then we obtain

$$
\begin{gathered}
f_{Q_{r}}\left|u_{t}(t, x)-\left(u_{t}\right)_{Q_{r}}\right|^{p} d x d t+f_{Q_{r}}\left|u_{x x^{\prime}}(t, x)-\left(u_{x x^{\prime}}\right)_{Q_{r}}\right|^{p} d x d t \\
\leq N \kappa^{d+2}\left(|f|^{p}\right)_{Q_{\kappa r}}+N \kappa^{-p}\left(\left|w_{x x}\right|^{p}+\left|w_{t}\right|^{p}+\lambda^{p / 2}\left|w_{x}\right|^{p}\right)_{Q_{\kappa r}} \\
+N \kappa^{-p}\left(\left|u_{x x}\right|^{p}+\left|u_{t}\right|^{p}+\lambda^{p / 2}\left|u_{x}\right|^{p}\right)_{Q_{\kappa r}} \\
\leq N \kappa^{d+2}\left(|f|^{p}\right)_{Q_{\kappa r}}+N \kappa^{-p}\left(\left|u_{x x}\right|^{p}+\left|u_{t}\right|^{p}+\lambda^{p / 2}\left|u_{x}\right|^{p}\right)_{Q_{\kappa r}} .
\end{gathered}
$$

To complete the proof, we use the fact that $u_{t}=f+\lambda u-a^{i j} u_{x^{i} x^{j}}$, and then let $\lambda \searrow 0$.

4. Proof of Theorem 3.2. Set

$$
L_{0} u=u_{t}+a^{i j}(t, x) u_{x^{i} x^{j}},
$$

where coefficients $a^{i j}$ satisfies Assumption 2.1 and 3.1.

Lemma 4.1. Let $p>q \geq 2$, and $r \in(0,1]$. Assume that $v \in W_{p, l o c}^{1,2}\left(\mathbb{R}^{d+1}\right)$ satisfies $L_{0} v=0$ in $Q_{2 r}$. Then

$$
\left(\left|v_{x x}\right|^{p}\right)_{Q_{r}}^{1 / p} \leq N\left(\left|v_{x x}\right|^{2}\right)_{Q_{2 r}}^{1 / 2} \leq N\left(\left|v_{x x}\right|^{q}\right)_{Q_{2 r}}^{1 / q}
$$

where $N$ depends only on $d, p, \delta$, and the function $\omega$.

Proof. This lemma is almost the same as Corollary 6.4 in [14] if $L_{0}$ is replaced by the operator used there. In our case, we can repeat the argument in Corollary 6.4 of $[14]$ if we have the estimate

$$
\left\|u_{x x}\right\|_{L_{p}\left(Q_{r}\right)} \leq N\left(\left\|L_{0} u\right\|_{L_{p}\left(Q_{\kappa r}\right)}+r^{-1}\left\|u_{x}\right\|_{L_{p}\left(Q_{\kappa r}\right)}+r^{-2}\|u\|_{L_{p}\left(Q_{\kappa r}\right)}\right)
$$

for $p \in(2, \infty)$ and $u \in W_{p, \text { loc }}^{1,2}\left(\mathbb{R}^{d+1}\right)$, where $r \in(0,1], \kappa \in(1, \infty)$, and $N$ depends only on $d, p, \delta, \kappa$, and the function $\omega$. This is obtained using Theorem 2.5 in [9] and the argument in the proof of Lemma 6.3 in [14].

The following theorem is proved in the same way as Lemma 3.1 in [14]. However, because of the different conditions on $a^{i j}$ between our operator $L_{0}$ and the operator defined in [14], we give a complete proof here.

THEOREM 4.2. Let $p \geq 2$. In case $p=2$, the coefficients $a^{i j}$ of $L_{0}$ are assumed to be independent of $x^{\prime} \in \mathbb{R}^{d-1}$. Then there exists a constant $N$, depending on $d, p$, $\delta$, and the function $\omega$, such that, for any $u \in C_{0}^{\infty}\left(\mathbb{R}^{d+1}\right), \kappa \geq 16$, and $r \in(0,1 / \kappa]$, we have

$$
f_{Q_{r}}\left|u_{t}(t, x)-\left(u_{t}\right)_{Q_{r}}\right|^{p} d x d t+f_{Q_{r}}\left|u_{x x^{\prime}}(t, x)-\left(u_{x x^{\prime}}\right)_{Q_{r}}\right|^{p} d x d t
$$




$$
\leq N \kappa^{d+2}\left(\left|L_{0} u\right|^{p}\right)_{Q_{\kappa r}}+N\left(\kappa^{-p}+\kappa^{d+2} \hat{a}^{1 / 2}\right)\left(\left|u_{x x}\right|^{p}\right)_{Q_{\kappa r}}
$$

where $\hat{a}=a_{\kappa r}^{\#\left(t, x^{\prime}\right)}$.

Proof. For given $u \in C_{0}^{\infty}\left(\mathbb{R}^{d+1}\right), \kappa \geq 16$, and $r \in(0,1 / \kappa]$, find a unique function $\tilde{w} \in W_{p}^{1,2}\left((-3,4) \times \mathbb{R}^{d}\right)$ satisfying $\tilde{w}(4, \bar{x})=0$ and

$$
L_{0} \tilde{w}=f I_{Q_{\kappa r}}
$$

where $f:=L_{0} u$. This is possible by Theorem 2.2 and 2.5 in [9]. Moreover, $\tilde{w} \in$ $W_{q}^{1,2}\left((-3,4) \times \mathbb{R}^{d}\right)$ for all $q \in(2, \infty)$ because $f I_{Q_{\kappa r}} \in L_{q}\left((-3,4) \times \mathbb{R}^{d}\right)$ for all $q>2$. Let

$$
w(t, x)=\eta(t) \tilde{w}(t, x)
$$

where $\eta(t)$ is an infinitely differentiable function defined on $\mathbb{R}$ such that

$$
\eta(t)=1, \quad-1 \leq t \leq 2, \quad \eta(t)=0, \quad t \leq-2 \quad \text { or } \quad t \geq 3
$$

We see that $w \in W_{p}^{1,2}\left(\mathbb{R}^{d+1}\right)$ and, in addition, $w \in W_{q}^{1,2}\left(\mathbb{R}^{d+1}\right)$ for all $q \in(2, \infty)$. From the estimates from Theorem 2.2 and 2.5 in [9] we have

$$
\int_{Q_{\kappa r}}\left(\left|w_{t}\right|^{p}+\left|w_{x x}\right|^{p}\right) d x d t \leq \int_{(-3,4) \times \mathbb{R}^{d}}\left(\left|\tilde{w}_{t}\right|^{p}+\left|\tilde{w}_{x x}\right|^{p}\right) d x d t \leq N \int_{Q_{\kappa r}}|f|^{p} d x d t
$$

where $N$ depends only on $d, \delta, p$, and the function $\omega$ (it also depends on the time interval, but the time interval here is fixed as $(-3,4))$. Thus

$$
\begin{gathered}
\left(\left|w_{t}\right|^{p}+\left|w_{x x}\right|^{p}\right)_{Q_{\kappa r}} \leq N\left(|f|^{p}\right)_{Q_{\kappa r}}, \\
\left(\left|w_{t}\right|^{p}+\left|w_{x x}\right|^{p}\right)_{Q_{r}} \leq N \kappa^{d+2}\left(|f|^{p}\right)_{Q_{\kappa r}} .
\end{gathered}
$$

where $N=N(d, \delta, p, \omega)$. and

Now we set $v=u-w$. Then $v \in W_{p}^{1,2}\left(\mathbb{R}^{d+1}\right), v \in W_{q}^{1,2}\left(\mathbb{R}^{d+1}\right)$ for all $q \in(2, \infty)$,

$$
L_{0} v=0 \quad \text { in } Q_{\kappa r}
$$

Let

$$
\bar{L}_{0}=\frac{\partial}{\partial t}+\bar{a}^{i j}\left(x^{1}\right) \frac{\partial^{2}}{\partial^{i} \partial^{j}}
$$

where

$$
\bar{a}^{i j}\left(x^{1}\right)=f_{0}^{(\kappa r / 2)^{2}} f_{B_{\kappa r / 2}^{\prime}} a^{i j}\left(s, x^{1}, y^{\prime}\right) d y^{\prime} d s
$$

Since $v \in W_{p}^{1,2}\left(\mathbb{R}^{d+1}\right)$ and $\kappa / 2 \geq 8$, by Theorem 3.9 applied to the operator $\bar{L}_{0}$, we have

$$
f_{Q_{r}}\left|v_{t}(t, x)-\left(v_{t}\right)_{Q_{r}}\right|^{p} d x d t+f_{Q_{r}}\left|v_{x x^{\prime}}(t, x)-\left(v_{x x^{\prime}}\right)_{Q_{r}}\right|^{p} d x d t
$$




$$
\leq N \kappa^{d+2}\left(\left|\bar{L}_{0} v\right|^{p}\right)_{Q_{\kappa r / 2}}+N \kappa^{-p}\left(\left|v_{x x}\right|^{p}\right)_{Q_{\kappa r / 2}} .
$$

Using the fact that $L_{0} v=0$ in $Q_{\kappa r}$, we have

$$
\begin{gathered}
\left(\left|\bar{L}_{0} v\right|^{p}\right)_{Q_{\kappa r / 2}}=f_{Q_{\kappa r / 2}}\left|\left(\bar{a}^{i j}\left(x^{1}\right)-a^{i j}(t, x)\right) v_{x^{i} x^{j}}\right|^{p} d x d t \\
\leq\left(f_{Q_{\kappa r / 2}}\left|\bar{a}^{i j}\left(x^{1}\right)-a^{i j}(t, x)\right|^{2 p} d x d t\right)^{1 / 2}\left(f_{Q_{\kappa r / 2}}\left|v_{x^{i} x^{j}}\right|^{2 p} d x d t\right)^{1 / 2},
\end{gathered}
$$

where we see

$$
f_{Q_{\kappa r / 2}}\left|\bar{a}^{i j}\left(x^{1}\right)-a^{i j}(t, x)\right|^{2 p} d x d t \leq N f_{Q_{\kappa r / 2}}\left|\bar{a}^{i j}\left(x^{1}\right)-a^{i j}(t, x)\right| d x d t \leq N a_{\kappa r / 2}^{\#\left(t, x^{\prime}\right)} .
$$

From Lemma 4.1 we also see

$$
\left(f_{Q_{\kappa r / 2}}\left|v_{x^{i} x^{j}}\right|^{2 p} d x d t\right)^{1 / 2} \leq N(d, p, \delta, \omega)\left(f_{Q_{\kappa r}}\left|v_{x x}\right|^{p} d x d t\right) .
$$

Hence

$$
\begin{gathered}
f_{Q_{r}}\left|v_{t}(t, x)-\left(v_{t}\right)_{Q_{r}}\right|^{p} d x d t+f_{Q_{r}}\left|v_{x x^{\prime}}(t, x)-\left(v_{x x^{\prime}}\right)_{Q_{r}}\right|^{p} d x d t \\
\leq N\left(\kappa^{-p}+\kappa^{d+2} \hat{a}^{1 / 2}\right)\left(\left|v_{x x}\right|^{p}\right)_{Q_{\kappa r}} .
\end{gathered}
$$

Note that

$$
\left(\left|v_{x x}\right|^{p}\right)_{Q_{\kappa r}} \leq N\left(\left|u_{x x}\right|^{p}\right)_{Q_{\kappa r}}+N\left(\left|w_{x x}\right|^{p}\right)_{Q_{\kappa r}} \leq N\left(\left|u_{x x}\right|^{p}\right)_{Q_{\kappa r}}+N\left(|f|^{p}\right)_{Q_{\kappa r}},
$$

where the second inequality is due to (10). Also note that, using the inequality (11),

$$
\begin{gathered}
f_{Q_{r}}\left|w_{x x^{\prime}}(t, x)-\left(w_{x x^{\prime}}\right)_{Q_{r}}\right|^{p} d x d t \leq N\left(\left|w_{x x^{\prime}}\right|^{p}\right)_{Q_{r}} \leq N \kappa^{d+2}\left(|f|^{p}\right)_{Q_{\kappa r}} \\
f_{Q_{r}}\left|w_{t}(t, x)-\left(w_{t}\right)_{Q_{r}}\right|^{p} d x d t \leq N\left(\left|w_{t}\right|^{p}\right)_{Q_{r}} \leq N \kappa^{d+2}\left(|f|^{p}\right)_{Q_{\kappa r}} .
\end{gathered}
$$

Therefore,

$$
\begin{gathered}
f_{Q_{r}}\left|u_{x x^{\prime}}(t, x)-\left(u_{x x^{\prime}}\right)_{Q_{r}}\right|^{p} d x d t \\
\leq N f_{Q_{r}}\left|v_{x x^{\prime}}(t, x)-\left(v_{x x^{\prime}}\right)_{Q_{r}}\right|^{p} d x d t+N f_{Q_{r}}\left|w_{x x^{\prime}}(t, x)-\left(w_{x x^{\prime}}\right)_{Q_{r}}\right|^{p} d x d t \\
\leq N\left(\kappa^{-p}+\kappa^{d+2} \hat{a}^{1 / 2}\right)\left(\left|u_{x x}\right|^{p}+|f|^{p}\right)_{Q_{\kappa r}}+N \kappa^{d+2}\left(|f|^{p}\right)_{Q_{\kappa r}} .
\end{gathered}
$$


Similarly, we have

$$
\begin{gathered}
f_{Q_{r}}\left|u_{t}(t, x)-\left(u_{t}\right)_{Q_{r}}\right|^{p} d x d t \\
\leq N\left(\kappa^{-p}+\kappa^{d+2} \hat{a}^{1 / 2}\right)\left(\left|u_{x x}\right|^{p}+|f|^{p}\right)_{Q_{\kappa r}}+N \kappa^{d+2}\left(|f|^{p}\right)_{Q_{\kappa r}} .
\end{gathered}
$$

The theorem is proved.

Here we introduce some notations we use below. Let

$$
\begin{array}{ll}
B_{r}^{d_{1}}\left(\mathbf{x}_{1}\right)=\left\{\left|\mathbf{x}_{1}-\mathbf{y}_{1}\right|<r: \mathbf{y}_{1} \in \mathbb{R}^{d_{1}}\right\}, & Q_{r}^{d_{1}}\left(t, \mathbf{x}_{1}\right)=\left(t, t+r^{2}\right) \times B_{r}^{d_{1}}\left(\mathbf{x}_{1}\right), \\
B_{r}^{d_{2}}\left(\mathbf{x}_{2}\right)=\left\{\left|\mathbf{x}_{2}-\boldsymbol{y}_{2}\right|<r: \mathbf{y}_{2} \in \mathbb{R}^{d_{2}}\right\}, & Q_{r}^{d_{2}}\left(t, \mathbf{x}_{2}\right)=\left(t, t+r^{2}\right) \times B_{r}^{d_{2}}\left(\mathbf{x}_{2}\right) .
\end{array}
$$

Recall that $\mathbf{x}_{1}$ and $\mathbf{x}_{2}$ are those defined in (3). As before, we set, for example, $B_{r}^{d_{1}}=B_{r}^{d_{1}}(0)$ and $Q_{r}^{d_{1}}=Q_{r}^{d_{1}}(0,0)$. For a function $f$ defined on $\mathbb{R}^{d+1}$, denote

$$
\left\|f\left(t, \mathbf{x}_{1}, \cdot\right)\right\|_{p\left(d_{2}\right)}=\left(\int_{\mathbb{R}^{d_{2}}}|f(t, x)|^{p} d \mathbf{x}_{2}\right)^{1 / p} .
$$

Note that $\left\|f\left(t, \mathbf{x}_{1}, \cdot\right)\right\|_{p\left(d_{2}\right)}$ is a function of $\left(t, \mathbf{x}_{1}\right)$. To define the above norm more precisely as well as explain some notations below, we add the following remark.

REMARK 4.3. As indicated in the introduction, we have fixed two nonnegative integers $d_{1}$ and $d_{2}\left(d_{1}+d_{2}=d\right)$ and all different integers $i_{1}, i_{2}, \cdots, i_{d_{1}}, j_{1}, j_{2}, \cdots, j_{d_{2}}$ from $\{1,2, \cdots, d\}$. For $x=\left(x^{1}, x^{2}, \cdots, x^{d}\right) \in \mathbb{R}^{d}$, we have denoted

$$
\mathbf{x}_{1}=\left(x^{i_{1}}, x^{i_{2}}, \cdots, x^{i_{d_{1}}}\right) \in \mathbb{R}^{d_{1}}, \quad \mathbf{x}_{2}=\left(x^{j_{1}}, x^{j_{2}}, \cdots, x^{j_{d_{2}}}\right) \in \mathbb{R}^{d_{2}} .
$$

Let $\Pi_{1}$ be a projection from $\mathbb{R}^{d}$ onto $\mathbb{R}^{d_{1}}$ such that $\Pi_{1}(x)=\mathbf{x}_{1}$. Likewise, let $\Pi_{2}$ be a projection from $\mathbb{R}^{d}$ onto $\mathbb{R}^{d_{2}}$ such that $\Pi_{2}(x)=\mathbf{x}_{2}$. Define $\Pi$ to be a function from $\mathbb{R}^{d}$ onto $\mathbb{R}^{d_{1}} \times \mathbb{R}^{d_{2}}$ such that $\Pi(x)=\left(\Pi_{1}(x), \Pi_{2}(x)\right)=\left(\mathbf{x}_{1}, \mathbf{x}_{2}\right)$. We see that the inverse $\Pi^{-1}$ of $\Pi$ exists. Then by $\left\|f\left(t, \mathbf{x}_{1}, \cdot\right)\right\|_{p\left(d_{2}\right)}$ we mean

$$
\left(\int_{\mathbb{R}^{d_{2}}}\left|f\left(t, \Pi^{-1}\left(\mathbf{x}_{1}, \mathbf{x}_{2}\right)\right)\right|^{p} d \mathbf{x}_{2}\right)^{1 / p} .
$$

In the following we use, for example, $u\left(t, \mathbf{x}_{1}, \mathbf{x}_{2}\right)$, the precise notation for which is therefore $u\left(t, \Pi^{-1}\left(\mathbf{x}_{1}, \mathbf{x}_{2}\right)\right)$.

LEMMA 4.4. Let $p \geq 2$. In case $p=2$, we assume that the coefficients $a^{i j}$ of $L_{0}$ are independent of $x^{\prime} \in \mathbb{R}^{d-1}$. Then there exists a constant $N=N\left(d_{1}, d_{2}, p, \delta, \omega\right)$ such that

$$
\begin{gathered}
f_{Q_{r}^{d_{1}}} f_{Q_{r}^{d_{1}}}\left|\left\|u_{t}\left(t, \mathbf{x}_{1}, \cdot\right)\right\|_{p\left(d_{2}\right)}-\left\|u_{t}\left(s, \mathbf{y}_{1}, \cdot\right)\right\|_{p\left(d_{2}\right)}\right|^{p} d \mathbf{x}_{1} d t d \mathbf{y}_{1} d s \\
+f_{Q_{r}^{d_{1}}} f_{Q_{r}^{d_{1}}}\left|\left\|u_{x x^{\prime}}\left(t, \mathbf{x}_{1}, \cdot\right)\right\|_{p\left(d_{2}\right)}-\left\|u_{x x^{\prime}}\left(s, \mathbf{y}_{1}, \cdot\right)\right\|_{p\left(d_{2}\right)}\right|^{p} d \mathbf{x}_{1} d t d \mathbf{y}_{1} d s
\end{gathered}
$$




$$
\begin{gathered}
\leq N\left(\kappa^{-p}+\kappa^{d+2} \hat{a}^{1 / 2}\right) f_{Q_{\kappa r}^{d_{1}}}\left\|u_{x x}\left(t, \mathbf{x}_{1}, \cdot\right)\right\|_{p\left(d_{2}\right)}^{p} d \mathbf{x}_{1} d t \\
\quad+N \kappa^{d+2} f_{Q_{\kappa r}^{d_{1}}}\left\|L_{0} u\left(t, \mathbf{x}_{1}, \cdot\right)\right\|_{p\left(d_{2}\right)}^{p} d \mathbf{x}_{1} d t
\end{gathered}
$$

for any $u \in C_{0}^{\infty}\left(\mathbb{R}^{d+1}\right), r>0$, and $\kappa \geq 16 \sqrt{2}$ satisfying $\kappa r \leq 1$, where $\hat{a}=a_{\kappa r}^{\#\left(t, x^{\prime}\right)}$.

Proof. Let us denote by $I$ the left-hand side of the inequality in the lemma. Note that

$$
\left|\left\|u_{t}\left(t, \mathbf{x}_{1}, \cdot\right)\right\|_{p\left(d_{2}\right)}-\left\|u_{t}\left(s, \mathbf{y}_{1}, \cdot\right)\right\|_{p\left(d_{2}\right)}\right|^{p} \leq\left\|u_{t}\left(t, \mathbf{x}_{1}, \cdot\right)-u_{t}\left(s, \mathbf{y}_{1}, \cdot\right)\right\|_{p\left(d_{2}\right)}^{p} .
$$

A similar inequality holds for the integrand of the second integral of $I$. Thus

$$
\begin{gathered}
I \leq f_{Q_{r}^{d_{1}}} f_{Q_{r}^{d_{1}}}\left\|u_{t}\left(t, \mathbf{x}_{1}, \cdot\right)-u_{t}\left(s, \mathbf{y}_{1}, \cdot\right)\right\|_{p\left(d_{2}\right)}^{p} d \mathbf{x}_{1} d t d \mathbf{y}_{1} d s \\
+f_{Q_{r}^{d_{1}}} f_{Q_{r}^{d_{1}}}\left\|u_{x x^{\prime}}\left(t, \mathbf{x}_{1}, \cdot\right)-u_{x x^{\prime}}\left(s, \mathbf{y}_{1}, \cdot\right)\right\|_{p\left(d_{2}\right)}^{p} d \mathbf{x}_{1} d t d \mathbf{y}_{1} d s:=I_{1}+I_{2} .
\end{gathered}
$$

Note that

$$
\begin{gathered}
\left\|u_{t}\left(t, \mathbf{x}_{1}, \cdot\right)-u_{t}\left(s, \mathbf{y}_{1}, \cdot\right)\right\|_{p\left(d_{2}\right)}^{p} \\
=f_{B_{r}^{d_{2}}} \int_{\mathbb{R}^{d_{2}}}\left|u_{t}\left(t, \mathbf{x}_{1}, \mathbf{z}_{2}+\mathbf{w}_{2}\right)-u_{t}\left(s, \mathbf{y}_{1}, \mathbf{z}_{2}+\mathbf{w}_{2}\right)\right|^{p} d \mathbf{z}_{2} d \mathbf{w}_{2} \\
=\int_{\mathbb{R}^{d_{2}}} f_{B_{r}^{d_{2}}\left(\mathbf{z}_{2}\right)}\left|u_{t}\left(t, \mathbf{x}_{1}, \mathbf{w}_{2}\right)-u_{t}\left(s, \mathbf{y}_{1}, \mathbf{w}_{2}\right)\right|^{p} d \mathbf{w}_{2} d \mathbf{z}_{2} .
\end{gathered}
$$

Hence

$$
I_{1}=\int_{\mathbb{R}^{d_{2}}} f_{Q_{r}^{d_{1}}} f_{Q_{r}^{d_{1}}} f_{B_{r}^{d_{2}}\left(\mathbf{z}_{2}\right)}\left|u_{t}\left(t, \mathbf{x}_{1}, \mathbf{w}_{2}\right)-u_{t}\left(s, \mathbf{y}_{1}, \mathbf{w}_{2}\right)\right|^{p} d \mathbf{w}_{2} d \mathbf{x}_{1} d t d \mathbf{y}_{1} d s d \mathbf{z}_{2} .
$$

Also note that

$$
\begin{gathered}
\left|u_{t}\left(t, \mathbf{x}_{1}, \mathbf{w}_{2}\right)-u_{t}\left(s, \mathbf{y}_{1}, \mathbf{w}_{2}\right)\right|^{p} \\
\leq 2^{p}\left|u_{t}\left(t, \mathbf{x}_{1}, \mathbf{w}_{2}\right)-\left(u_{t}\right)_{Q_{\sqrt{2} r}\left(0, \mathbf{z}_{2}\right)}\right|^{p}+2^{p}\left|u_{t}\left(s, \mathbf{y}_{1}, \mathbf{w}_{2}\right)-\left(u_{t}\right)_{Q_{\sqrt{2} r}\left(0, \mathbf{z}_{2}\right)}\right|^{p}
\end{gathered}
$$

and

$$
Q_{r}^{d_{1}} \times B_{r}^{d_{2}}\left(\mathbf{z}_{2}\right) \subset Q_{\sqrt{2} r}\left(0, \mathbf{z}_{2}\right),
$$

where, and throughout the proof, $\left(0, z_{2}\right)=\left(0, \Pi^{-1}\left(0, z_{2}\right)\right)$ (see Remark 4.3). Thus

$$
I_{1} \leq N(d, p) \int_{\mathbb{R}^{d_{2}}} f_{Q_{\sqrt{2} r}\left(0, \mathbf{z}_{2}\right)}\left|u_{t}\left(t, \mathbf{x}_{1}, \mathbf{w}_{2}\right)-\left(u_{t}\right)_{Q_{\sqrt{2} r}\left(0, \mathbf{z}_{2}\right)}\right|^{p} d \mathbf{w}_{2} d \mathbf{x}_{1} d t d \mathbf{z}_{2} .
$$


Then by Theorem 4.2 (with an appropriate translation) we have

$$
\begin{gathered}
I_{1} \leq N \kappa^{d+2} \int_{\mathbb{R}^{d_{2}}}\left(|f|^{p}\right)_{Q_{\kappa r}\left(0, \mathbf{z}_{2}\right)} d \mathbf{z}_{2} \\
+N\left(\kappa^{-p}+\kappa^{d+2}\left(a_{\kappa r}^{\#\left(t, x^{\prime}\right)}\right)^{1 / 2}\right) \int_{\mathbb{R}^{d_{2}}}\left(\left|u_{x x}\right|^{p}\right)_{Q_{\kappa r}\left(0, \mathbf{z}_{2}\right)} d \mathbf{z}_{2},
\end{gathered}
$$

where $f=L_{0} u, \kappa \geq 16 \sqrt{2}$, and $\kappa r \leq 1$. Observe that

$$
\begin{gathered}
\int_{\mathbb{R}^{d_{2}}}\left(|f|^{p}\right)_{Q_{\kappa r}\left(0, \mathbf{z}_{2}\right)} d \mathbf{z}_{2}=\int_{\mathbb{R}^{d_{2}}} f_{Q_{\kappa r}}\left|f\left(t, \mathbf{x}_{1}, \mathbf{z}_{2}+\mathbf{w}_{2}\right)\right|^{p} d \mathbf{w}_{2} d \mathbf{x}_{1} d t d \mathbf{z}_{2} \\
\leq N(d) \int_{\mathbb{R}^{d_{2}}} f_{Q_{\kappa r}^{d_{1}}} f_{B_{\kappa r}^{d_{2}}}\left|f\left(t, \mathbf{x}_{1}, \mathbf{z}_{2}+\mathbf{w}_{2}\right)\right|^{p} d \mathbf{w}_{2} d \mathbf{x}_{1} d t d \mathbf{z}_{2} \\
=N(d) f_{Q_{\kappa r}^{d_{1}}} \int_{\mathbb{R}^{d_{2}}}\left|f\left(t, \mathbf{x}_{1}, \mathbf{z}_{2}\right)\right|^{p} d \mathbf{z}_{2} d \mathbf{x}_{1} d t=N(d) f_{Q_{\kappa r}^{d_{1}}}\left\|f\left(t, \mathbf{x}_{1}, \cdot\right)\right\|_{p\left(d_{2}\right)}^{p} d \mathbf{x}_{1} d t .
\end{gathered}
$$

Similarly,

$$
\int_{\mathbb{R}^{d_{2}}}\left(\left|u_{x x}\right|^{p}\right)_{Q_{\kappa r}\left(0, \mathbf{z}_{2}\right)} d \mathbf{z}_{2} \leq N(d) f_{Q_{\kappa r}^{d_{1}}}\left\|u_{x x}\left(t, \mathbf{x}_{1}, \cdot\right)\right\|_{p\left(d_{2}\right)}^{p} d \mathbf{x}_{1} d t
$$

Therefore,

$$
\begin{gathered}
I_{1} \leq N \kappa^{d+2} f_{Q_{\kappa r}^{d_{1}}}\left\|f\left(t, \mathbf{x}_{1}, \cdot\right)\right\|_{p\left(d_{2}\right)}^{p} d \mathbf{x}_{1} d t \\
+N\left(\kappa^{-p}+\kappa^{d+2}\left(a_{\kappa r}^{\#\left(t, x^{\prime}\right)}\right)^{1 / 2}\right) f_{Q_{\kappa r}^{d_{1}}}\left\|u_{x x}\left(t, \mathbf{x}_{1}, \cdot\right)\right\|_{p\left(d_{2}\right)}^{p} d \mathbf{x}_{1} d t .
\end{gathered}
$$

By following the same argument above, we arrive at the above inequality with $I_{2}$ in place of $I_{1}$. This finishes the proof.

Set

$$
\begin{gathered}
\phi\left(t, \mathbf{x}_{1}\right)=\left\|u_{t}\left(t, \mathbf{x}_{1}, \cdot\right)\right\|_{p\left(d_{2}\right)}, \quad \varphi\left(t, \mathbf{x}_{1}\right)=\left\|u_{x x^{\prime}}\left(t, \mathbf{x}_{1}, \cdot\right)\right\|_{p\left(d_{2}\right)}, \\
\zeta\left(t, \mathbf{x}_{1}\right)=\left\|u_{x x}\left(t, \mathbf{x}_{1}, \cdot\right)\right\|_{p\left(d_{2}\right)}, \quad \psi\left(t, \mathbf{x}_{1}\right)=\left\|L_{0} u\left(t, \mathbf{x}_{1}, \cdot\right)\right\|_{p\left(d_{2}\right)} .
\end{gathered}
$$

Then they are functions of $\left(t, \mathbf{x}_{1}\right) \in \mathbb{R} \times \mathbb{R}^{d_{1}}$. Let $\mathbb{Q}^{d_{1}}$ be the collection of all $Q_{r}^{d_{1}}\left(t, \mathbf{x}_{1}\right)$, $\left(t, \mathbf{x}_{1}\right) \in \mathbb{R} \times \mathbb{R}^{d_{1}}, r \in(0, \infty)$. The maximal and sharp function of $g\left(t, \mathbf{x}_{1}\right),\left(t, \mathbf{x}_{1}\right) \in$ $\mathbb{R} \times \mathbb{R}^{d_{1}}$, are defined by

$$
\begin{gathered}
M g\left(t, \mathbf{x}_{1}\right)=\sup _{\left(t, \mathbf{x}_{1}\right) \in Q^{d_{1}}} f_{Q^{d_{1}}}\left|g\left(s, \mathbf{y}_{1}\right)\right| d \mathbf{y}_{1} d s, \\
g^{\#}\left(t, \mathbf{x}_{1}\right)=\sup _{\left(t, \mathbf{x}_{1}\right) \in Q^{d_{1}}} f_{Q^{d_{1}}}\left|g\left(s, \mathbf{y}_{1}\right)-(g)_{Q^{d_{1}}}\right| d \mathbf{y}_{1} d s,
\end{gathered}
$$


where the supremums are taken over all $Q^{d_{1}} \in \mathbb{Q}^{d_{1}}$ containing $\left(t, \mathbf{x}_{1}\right)$.

Lemma 4.5. Let $p \geq 2$. In case $p=2$, we assume that the coefficients $a^{i j}$ of $L_{0}$ are independent of $x^{\prime} \in \mathbb{R}^{d-1}$. Let $R \in(0,1]$ and $u$ be a function in $C_{0}^{\infty}\left(\mathbb{R}^{d+1}\right)$ such that

$$
u(t, x)=u\left(t, \mathbf{x}_{1}, \mathbf{x}_{2}\right)=0 \quad \text { if }\left(t, \mathbf{x}_{1}\right) \notin\left(0, R^{4}\right) \times B_{R^{2}}^{d_{1}} .
$$

Then

$$
\begin{gathered}
\phi^{\#}\left(t, \mathbf{x}_{1}\right)+\varphi^{\#}\left(t, \mathbf{x}_{1}\right) \leq N \kappa^{(d+2) / p}\left(M \psi^{p}\left(t, \mathbf{x}_{1}\right)\right)^{1 / p}+N(\kappa R)^{2-2 / p}\left(M \phi^{p}\left(t, \mathbf{x}_{1}\right)\right)^{1 / p} \\
+N\left((\kappa R)^{\left(d_{1}+2\right)(1-1 / p)}+\kappa^{-1}+\kappa^{(d+2) / p}(\omega(R))^{1 /(2 p)}\right)\left(M \zeta^{p}\left(t, \mathbf{x}_{1}\right)\right)^{1 / p}
\end{gathered}
$$

for all $\kappa \geq 16 \sqrt{2}$ and $\left(t, \mathbf{x}_{1}\right) \in \mathbb{R} \times \mathbb{R}^{d_{1}}$, where $N=N\left(d_{1}, d_{2}, p, \delta, \omega\right)$.

Proof. Take a $\kappa$ such that $\kappa \geq 16 \sqrt{2}$. If $r \leq R / \kappa$, then

$$
\kappa r \leq R \leq 1, \quad a_{\kappa r}^{\#\left(t, x^{\prime}\right)} \leq a_{R}^{\#\left(t, x^{\prime}\right)} \leq \omega(R) .
$$

Thus by Lemma 4.4,

$$
\begin{gathered}
f_{Q_{r}^{d_{1}}}\left|\phi\left(s, \boldsymbol{y}_{1}\right)-(\phi)_{Q_{r}^{d_{1}}}\right|^{p} d \mathbf{y}_{1} d s+f_{Q_{r}^{d_{1}}}\left|\varphi\left(s, \mathbf{y}_{1}\right)-(\varphi)_{Q_{r}^{d_{1}}}\right|^{p} d \mathbf{y}_{1} d s \\
\leq N \kappa^{d+2}\left(\psi^{p}\right)_{Q_{\kappa r}^{d_{1}}}+N\left(\kappa^{-p}+\kappa^{d+2}(\omega(R))^{1 / 2}\right)\left(\phi^{p}\right)_{Q_{\kappa r}^{d_{1}} .}
\end{gathered}
$$

From this and an appropriate translation we obtain, for $\left(\bar{t}, \overline{\mathbf{x}}_{1}\right) \in \mathbb{R} \times \mathbb{R}^{d_{1}}$,

$$
\begin{gathered}
f_{Q_{r}^{d_{1}\left(\bar{t}, \overline{\mathbf{x}}_{1}\right)}}\left|\phi\left(s, \boldsymbol{y}_{1}\right)-(\phi)_{Q_{r}^{d_{1}\left(\bar{t}, \overline{\mathbf{x}}_{1}\right)}}\right|^{p} d \mathbf{y}_{1} d s+f_{Q_{r}^{d_{1}\left(\bar{t}, \overline{\mathbf{x}}_{1}\right)}}\left|\varphi\left(s, \boldsymbol{y}_{1}\right)-(\varphi)_{Q_{r}^{d_{1}\left(\bar{t}, \overline{\mathbf{x}}_{1}\right)}}\right|^{p} d \mathbf{y}_{1} d s \\
\leq N \kappa^{d+2}\left(\psi^{p}\right)_{Q_{\kappa r}^{d_{1}\left(\bar{t}, \overline{\mathbf{x}}_{1}\right)}}+N\left(\kappa^{-p}+\kappa^{d+2}(\omega(R))^{1 / 2}\right)\left(\zeta^{p}\right)_{Q_{\kappa r}^{d_{1}\left(\bar{t}, \overline{\mathbf{x}}_{1}\right)}}
\end{gathered}
$$

if $r \leq R / \kappa$. Then using the Hölder's inequality it follows that, for $r \leq R / \kappa$,

$$
\begin{gathered}
f_{Q_{r}^{d_{1}\left(\bar{t}, \overline{\mathbf{x}}_{1}\right)}}\left|\phi\left(s, \mathbf{y}_{1}\right)-(\phi)_{Q_{r}^{d_{1}\left(\bar{t}, \overline{\mathbf{x}}_{1}\right)}}\right| d \mathbf{y}_{1} d s+f_{Q_{r}^{d_{1}\left(\bar{t}, \overline{\mathbf{x}}_{1}\right)}}\left|\varphi\left(s, \mathbf{y}_{1}\right)-(\varphi)_{Q_{r}^{d_{1}\left(\bar{t}, \overline{\mathbf{x}}_{1}\right)}}\right| d \mathbf{y}_{1} d s \\
\leq N \kappa^{(d+2) / p}\left(\psi^{p}\right)_{Q_{\kappa r}^{d_{1}\left(\bar{t}, \overline{\mathbf{x}}_{1}\right)}}^{1 / p}+N\left(\kappa^{-1}+\kappa^{(d+2) / p}(\omega(R))^{1 /(2 p)}\right)\left(\zeta^{p}\right)_{Q_{\kappa r}^{d_{1}\left(\bar{t}, \overline{\mathbf{x}}_{1}\right)}}^{1 / p} .
\end{gathered}
$$

On the other hand, if $r>R / \kappa$,

$$
\begin{gathered}
f_{Q_{r}^{d_{1}\left(\bar{t}, \overline{\mathbf{x}}_{1}\right)}}\left|\phi\left(s, \mathbf{y}_{1}\right)-(\phi)_{Q_{r}^{d_{1}\left(\bar{t}, \overline{\mathbf{x}}_{1}\right)}}\right| d \boldsymbol{y}_{1} d s \\
\leq 2\left(f_{Q_{r}^{d_{1}\left(\bar{t}, \overline{\mathbf{x}}_{1}\right)}} I_{Q_{R^{2}}^{d_{1}}} d \boldsymbol{y}_{1} d s\right)^{1-1 / p}\left(f_{Q_{r}^{d_{1}\left(\bar{t}, \overline{\mathbf{x}}_{1}\right)}}\left|\phi\left(s, \boldsymbol{y}_{1}\right)\right|^{p} d \boldsymbol{y}_{1} d s\right)^{1 / p}
\end{gathered}
$$




$$
\begin{aligned}
& \leq N\left(d_{1}\right)\left(R^{2} / r\right)^{\left(d_{1}+2\right)(1-1 / p)}\left(\phi^{p}\right)_{Q_{r}^{d_{1}}\left(\bar{t}, \overline{\mathbf{x}}_{1}\right)}^{1 / p} \\
& \leq N\left(d_{1}\right)(\kappa R)^{\left(d_{1}+2\right)(1-1 / p)}\left(\phi^{p}\right)_{Q_{r}^{d_{1}}\left(\bar{t}, \overline{\mathbf{x}}_{1}\right)}^{1 / p} .
\end{aligned}
$$

By the same reasoning, if $r>R / \kappa$, we have

$$
\int_{Q_{r}^{d_{1}}\left(\bar{t}, \overline{\mathbf{x}}_{1}\right)}\left|\varphi\left(s, \mathbf{y}_{1}\right)-(\varphi)_{Q_{r}^{d_{1}}\left(\bar{t}, \overline{\mathbf{x}}_{1}\right)}\right| d \mathbf{y}_{1} d s \leq N(\kappa R)^{\left(d_{1}+2\right)(1-1 / p)}\left(\varphi^{p}\right)_{Q_{r}^{d_{1}}\left(\bar{t}_{,}, \overline{\mathbf{x}}_{1}\right)}^{1 / p}
$$

Therefore,

$$
\begin{gathered}
\int_{Q_{r}^{d_{1}\left(\bar{t}, \overline{\mathbf{x}}_{1}\right)}}\left|\phi\left(s, \mathbf{y}_{1}\right)-(\phi)_{Q_{r}^{d_{1}}\left(\bar{t}, \overline{\mathbf{x}}_{1}\right)}\right| d \mathbf{y}_{1} d s+f_{Q_{r}^{d_{1}\left(\bar{t}, \overline{\mathbf{x}}_{1}\right)}}\left|\varphi\left(s, \mathbf{y}_{1}\right)-(\varphi)_{Q_{r}^{d_{1}}\left(\bar{t}, \overline{\mathbf{x}}_{1}\right)}\right| d \mathbf{y}_{1} d s \\
\leq N \kappa^{(d+2) / p}\left(\psi^{p}\right)_{Q_{\kappa r}^{d_{1}\left(\bar{t}, \overline{\mathbf{x}}_{1}\right)}}^{1 / p}+N\left(\kappa^{-1}+\kappa^{(d+2) / p}(\omega(R))^{1 /(2 p)}\right)\left(\zeta^{p}\right)_{Q_{\kappa r}^{d_{1}\left(\bar{t}, \overline{\mathbf{x}}_{1}\right)}}^{1 / p} \\
+N(\kappa R)^{\left(d_{1}+2\right)(1-1 / p)}\left(\left(\phi^{p}\right)_{Q_{r}^{d_{1}\left(\bar{t}, \overline{\mathbf{x}}_{1}\right)}}^{1 / p}+\left(\varphi^{p}\right)_{\left.Q_{r}^{d_{1}\left(\bar{t}, \overline{\mathbf{x}}_{1}\right)}\right)}^{1 / p}\right)
\end{gathered}
$$

for all $r>0$.

Now we observe that, for any $\left(t, \mathbf{x}_{1}\right) \in Q_{r}\left(\bar{t}, \overline{\mathbf{x}}_{1}\right)$,

$$
\begin{aligned}
& \left(\psi^{p}\right)_{Q_{\kappa r}^{d_{1}\left(\bar{t}, \overline{\mathbf{x}}_{1}\right)}} \leq M \psi^{p}\left(t, \mathbf{x}_{1}\right), \quad\left(\zeta^{p}\right)_{Q_{\kappa r}^{d_{1}\left(\bar{t}, \overline{\mathbf{x}}_{1}\right)}} \leq M \zeta^{p}\left(t, \mathbf{x}_{1}\right), \\
& \left(\phi^{p}\right)_{Q_{\kappa r}^{d_{1}}\left(\bar{t}, \overline{\mathbf{x}}_{1}\right)} \leq M \phi^{p}\left(t, \mathbf{x}_{1}\right), \quad\left(\varphi^{p}\right)_{Q_{\kappa r}^{d_{1}\left(\bar{t}, \overline{\mathbf{x}}_{1}\right)}} \leq M \zeta^{p}\left(t, \mathbf{x}_{1}\right) .
\end{aligned}
$$

The last two inequalities also hold true if $\kappa r$ is replaced by $r$. From these inequalities as well as (12) it follows that, for any $Q^{d_{1}} \in \mathbb{Q}^{d_{1}}$ such that $Q^{d_{1}} \ni\left(t, \mathbf{x}_{1}\right)$,

$$
\begin{aligned}
& f_{Q^{d_{1}}}\left|\phi\left(s, \boldsymbol{y}_{1}\right)-(\phi)_{Q^{d_{1}}}\right| d \boldsymbol{y}_{1} d s+f_{Q^{d_{1}}}\left|\varphi\left(s, \mathbf{y}_{1}\right)-(\varphi)_{Q^{d_{1}}}\right| d \mathbf{y}_{1} d s \\
& \leq N \kappa^{(d+2) / p}\left(M \psi^{p}\left(t, \mathbf{x}_{1}\right)\right)^{1 / p}+N(\kappa R)^{\left(d_{1}+2\right)(1-1 / p)}\left(M \phi^{p}\left(t, \mathbf{x}_{1}\right)\right)^{1 / p} \\
&+N\left((\kappa R)^{\left(d_{1}+2\right)(1-1 / p)}+\kappa^{-1}+\kappa^{(d+2) / p}(\omega(R))^{1 /(2 p)}\right)\left(M \zeta^{p}\left(t, \mathbf{x}_{1}\right)\right)^{1 / p} .
\end{aligned}
$$

Taking the supremum of the left-hand side of the above inequality over all $Q^{d_{1}} \in \mathbb{Q}^{d_{1}}$ such that $Q^{d_{1}} \ni\left(t, \mathbf{x}_{1}\right)$, we obtain the inequality in the lemma. The lemma is proved.

Now we set

$$
\|u\|_{L_{q, p}}=\|u\|_{L_{q}^{t, \mathbf{x}_{1}} L_{p}^{\mathrm{x}_{2}}\left(\mathbb{R} \times \mathbb{R}^{d}\right)} .
$$

COROLlary 4.6. Let $q>p \geq 2$. In case $p=2$, we assume that the coefficients $a^{i j}$ of $L_{0}$ are independent of $x^{\prime} \in \mathbb{R}^{\bar{d}-1}$. Then there exist constants $R$ and $N$, depending only on $d_{1}, d_{2}, p, q, \delta$, and the function $\omega$, such that

$$
\left\|u_{t}\right\|_{L_{q, p}}+\left\|u_{x x}\right\|_{L_{q, p}} \leq N\left\|L_{0} u\right\|_{L_{q, p}}
$$


for any $u \in C_{0}^{\infty}\left(\mathbb{R}^{d+1}\right)$ satisfying

$$
u(t, x)=u\left(t, \mathbf{x}_{1}, \mathbf{x}_{2}\right)=0 \quad \text { if }\left(t, \mathbf{x}_{1}\right) \notin\left(0, R^{4}\right) \times B_{R^{2}}^{d_{1}} .
$$

Proof. Let $u \in C_{0}^{\infty}\left(\mathbb{R}^{d+1}\right)$ be a function such that

$$
u(t, x)=u\left(t, \mathbf{x}_{1}, \mathbf{x}_{2}\right)=0 \quad \text { if }\left(t, x^{\prime}\right) \notin\left(0, R^{4}\right) \times B_{R^{2}}^{d_{1}},
$$

where $R \in(0,1]$ will be specified below. Using the inequality in Lemma 4.5 as well as the Hardy-Littlewood theorem and Fefferman-Stein theorem (note that $q / p>1$ ), we have

$$
\begin{gathered}
\left\|u_{t}\right\|_{L_{q, p}}+\left\|u_{x x^{\prime}}\right\|_{L_{q, p}} \leq N \kappa^{(d+2) / p}\left\|L_{0} u\right\|_{L_{q, p}}+N(\kappa R)^{2-2 / p}\left\|u_{t}\right\|_{L_{q, p}} \\
+N\left((\kappa R)^{\left(d_{1}+2\right)(1-1 / p)}+\kappa^{-1}+\kappa^{(d+2) / p}(\omega(R))^{1 /(2 p)}\right)\left\|u_{x x}\right\|_{L_{q, p}} .
\end{gathered}
$$

for all $\kappa \geq 16 \sqrt{2}$. The left-hand side of the above inequality can be replaced by $\left\|u_{t}\right\|_{L_{q, p}}+\left\|u_{x x}\right\|_{L_{q, p}}$ because

$$
u_{x^{1} x^{1}}=\frac{1}{a^{11}}\left(L_{0} u-u_{t}-\sum_{i \neq 1 \text { or } j \neq 1} a^{i j} u_{x^{1} x^{j}}\right) .
$$

Now we choose a large $\kappa$ and then a small $R$ so that

$$
N\left((\kappa R)^{\left(d_{1}+2\right)(1-1 / p)}+\kappa^{-1}+\kappa^{(d+2) / p}(\omega(R))^{1 /(2 p)}\right)<1 / 2
$$

and

$$
N(\kappa R)^{2-2 / p}<1 / 2
$$

It then follows that

$$
\left\|u_{t}\right\|_{L_{q, p}}+\left\|u_{x x}\right\|_{L_{q, p}} \leq 2 N \kappa^{(d+2) / p}\left\|L_{0} u\right\|_{L_{q, p}} .
$$

This finishes the proof.

REMARK 4.7. In the above corollary the estimate is prove when $L_{q, p}=$ $L_{q}^{t, \mathbf{x}_{1}} L_{p}^{\mathbf{X}_{2}}\left(\mathbb{R} \times \mathbb{R}^{d}\right)$. However, by making appropriates changes in Lemma 4.4 and Lemma 4.5, we prove the same estimate when $L_{q, p}=L_{q}^{\mathbf{x}_{1}} L_{p}^{t, \mathbf{x}_{2}}\left(\mathbb{R} \times \mathbb{R}^{d}\right)$.

TheOREM 4.8. Under the assumptions on $L$ given in Theorem 3.2, there exist $\lambda_{0} \geq 0$ and $N$, depending only on $d_{1}, d_{2}, \delta, K, p, q$, and the function $\omega$, such that

$$
\begin{aligned}
\lambda\|u\|_{L_{q, p}\left((T, \infty) \times \mathbb{R}^{d}\right)}+\sqrt{\lambda} \| & u_{x}\left\|_{L_{q, p}\left((T, \infty) \times \mathbb{R}^{d}\right)}+\right\| u_{x x} \|_{L_{q, p}\left((T, \infty) \times \mathbb{R}^{d}\right)} \\
+\left\|u_{t}\right\|_{L_{q, p}\left((T, \infty) \times \mathbb{R}^{d}\right)} \leq N\|(L-\lambda) u\|_{L_{q, p}\left((T, \infty) \times \mathbb{R}^{d}\right)} &
\end{aligned}
$$

for all $\lambda \geq \lambda_{0}$ and $u \in W_{q, p}^{1,2}\left((T, \infty) \times \mathbb{R}^{d}\right)$, where $-\infty \leq T<\infty$.

Proof. By the argument following Corollary 5.14 in [14] it is enough to prove (14) for $T=-\infty$. Moreover, it is enough to prove (14) for $u \in C_{0}^{\infty}\left(\mathbb{R}^{d+1}\right)$. Then the inequality (14) follows from Corollary 4.6 (also see Remark 4.7) and a partition of unity. Details can be obtained by following the proofs in section 3 [14], more precisely, those of Lemma 3.4 and Theorem 3.5 in [14].

Proof of Theorem 3.2. The estimate in Theorem 4.8 along with the method of continuity implies Theorem 3.2. For details, see the proof of Theorem 2.1 in [13]. 
5. Equations with $a^{i j}$ measurable in $\left(t, x^{1}\right) \in \mathbb{R}^{2}$. Throughout this section, we set

$$
\mathfrak{L}_{\lambda} u=u_{t}+a^{i j}\left(t, x^{1}\right) u_{x^{i} x^{j}}-\lambda u,
$$

where $\lambda \geq 0$ and $a^{i j}$ are functions of only $\left(t, x^{1}\right) \in \mathbb{R}^{2}, a^{11}\left(x^{1}\right)$ is a function of $x^{1} \in \mathbb{R}$, satisfying Assumption 2.1.

As is seen in [7], one of key steps there is based on Theorem 3.2 in this paper when $d_{1}=0$ and $d_{2}=d$. Now that we have proved Theorem 3.2, it is legitimate to use the results in [7]. Thus we can state the following theorem, which is due to the result in [7] as well as in [9].

TheOrem 5.1. Let $p \geq 2$ and $T \in[-\infty, \infty)$. For any $\lambda>0$ and $f \in L_{p}((T, \infty) \times$ $\left.\mathbb{R}^{d}\right)$, there exists a unique solution $u \in W_{p}^{1,2}\left((T, \infty) \times \mathbb{R}^{d}\right)$ to the equation $\mathfrak{L}_{\lambda} u=f$. Furthermore, there is a constant $N=N(d, p, \delta)$ such that, for any $\lambda \geq 0$ and $u \in$ $W_{p}^{1,2}\left((T, \infty) \times \mathbb{R}^{d}\right)$, we have

$$
\begin{gathered}
\left\|u_{t}\right\|_{L_{p}\left((T, \infty) \times \mathbb{R}^{d}\right)}+\left\|u_{x x}\right\|_{L_{p}\left((T, \infty) \times \mathbb{R}^{d}\right)}+\sqrt{\lambda}\left\|u_{x}\right\|_{L_{p}\left((T, \infty) \times \mathbb{R}^{d}\right)} \\
+\lambda\|u\|_{L_{p}\left((T, \infty) \times \mathbb{R}^{d}\right)} \leq N\left\|\mathfrak{L}_{\lambda} u\right\|_{L_{p}\left((T, \infty) \times \mathbb{R}^{d}\right)} .
\end{gathered}
$$

More precisely, this theorem follows, in case $p=2$, from Theorem 3.2 in [9] and, in case $p>2$, from Corollary 4.2 in [7] as well as the argument in the proof of Theorem 4.1 in [13] (also see the discussion following Theorem 3.3).

Based on the estimate in the above theorem, we have the following lemma, the proof of which is almost identical to those of Lemma 3.4 and Lemma 5.3 (a complete proof of Lemma 5.3 is given). Recall that $\Lambda_{r}=\left(0, r^{2}\right) \times(-r, r) \times B_{r}^{\prime}$.

Lemma 5.2. Let $p \in[2, \infty)$. For any $u \in W_{p, l o c}^{1,2}\left(\mathbb{R}^{d+1}\right)$, we have

$$
\left\|u_{t}\right\|_{L_{p}\left(\Lambda_{r}\right)}+\left\|u_{x x}\right\|_{L_{p}\left(\Lambda_{r}\right)}+\left\|u_{x}\right\|_{L_{p}\left(\Lambda_{r}\right)} \leq N\left(\left\|\mathfrak{L}_{0} u\right\|_{L_{p}\left(\Lambda_{R}\right)}+\|u\|_{L_{p}\left(\Lambda_{R}\right)}\right),
$$

where $0<r<R<\infty$ and $N=N(d, p, \delta, r, R)$.

In the following we set $\|u\|_{L_{q, p}\left(\Lambda_{r}\right)}=\|u\|_{L_{q}^{t, x^{1}} L_{p}^{x^{\prime}}\left(\Lambda_{r}\right)}$, i.e.,

$$
\|u\|_{L_{q, p}\left(\Lambda_{r}\right)}=\left(\int_{0}^{r^{2}} \int_{-r}^{r}\left(\int_{B_{r}^{\prime}}\left|u\left(t, x^{1}, x^{\prime}\right)\right|^{p} d x^{\prime}\right)^{q / p} d x^{1} d t\right)^{1 / q} .
$$

Lemma 5.3. Let

$$
\mathcal{L}_{0} u=u_{t}+a^{11}\left(x^{1}\right) u_{x^{1} x^{1}}+\Delta_{d-1} u,
$$

where $\Delta_{d-1} u=\sum_{j=2}^{d} u_{x^{j} x^{j}}$. Then for $p, q \in[2, \infty)$ such that $q \geq p$, we have

$$
\left\|u_{t}\right\|_{L_{q, p}\left(\Lambda_{r}\right)}+\left\|u_{x x}\right\|_{L_{q, p}\left(\Lambda_{r}\right)}+\left\|u_{x}\right\|_{L_{q, p}\left(\Lambda_{r}\right)} \leq N\left(\left\|\mathcal{L}_{0} u\right\|_{L_{q, p}\left(\Lambda_{R}\right)}+\|u\|_{L_{q, p}\left(\Lambda_{R}\right)}\right)
$$

for $u \in C_{l o c}^{\infty}\left(\mathbb{R}^{d+1}\right)$, where $0<r<R<\infty$ and $N=N(d, p, q, \delta, r, R)$. 
Proof. The case $p=q$ is proved in Lemma 5.2, so we assume that $q>p$. Set

$$
\begin{aligned}
& r_{0}=r, \quad r_{m}=r+(R-r) \sum_{k=1}^{m} \frac{1}{2^{k}}, \quad m=1,2, \cdots, \\
& \Lambda_{m}=\left(0, r_{m}\right)^{2} \times\left(-r_{m}, r_{m}\right) \times B_{r_{m}}^{\prime}, \quad m=0,1, \cdots .
\end{aligned}
$$

Then take $\eta_{m} \in C_{0}^{\infty}\left(\mathbb{R}^{d+1}\right)$ such that

$$
\begin{aligned}
& \eta_{m}(t, x)= \begin{cases}1 & \text { if } \quad(t, x) \in \Lambda_{m}, \\
0 & \text { if } \quad(t, x) \notin\left(-r_{m+1}^{2}, r_{m+1}^{2}\right) \times\left(-r_{m+1}, r_{m+1}\right) \times B_{r_{m+1}}^{\prime},\end{cases} \\
& \left|\left(\eta_{m}\right)_{x}\right| \leq N \frac{2^{m+1}}{R-r}, \quad\left|\left(\eta_{m}\right)_{x x}\right| \leq N \frac{2^{2 m+2}}{(R-r)^{2}}, \quad\left|\left(\eta_{m}\right)_{t}\right| \leq N \frac{2^{2 m+2}}{(R-r)^{2}},
\end{aligned}
$$

where $N$ depends only on $d$. To construct them take an infinitely differentiable function $g(s), s \in(-\infty, \infty)$, such that $g(s)=1$ for $s \leq 0, g(s)=0$ for $s \geq 1$, and $0 \leq g \leq 1$. After this define

$$
\eta_{m}\left(t, x^{1}, x^{\prime}\right)=\eta_{1 m}(t) \eta_{2 m}\left(x^{1}\right) \eta_{3 m}\left(x^{\prime}\right),
$$

where

$$
\begin{gathered}
\eta_{1_{m}}(t)=g\left(2^{m+1}(R-r)^{-1}\left(\sqrt{|t|}-r_{m}\right)\right), \eta_{2 m}\left(x^{1}\right)=g\left(2^{m+1}(R-r)^{-1}\left(\left|x^{1}\right|-r_{m}\right)\right), \\
\eta_{3_{m}}\left(x^{\prime}\right)=g\left(2^{m+1}(R-r)^{-1}\left(\left|x^{\prime}\right|-r_{m}\right)\right) .
\end{gathered}
$$

Now we observe that the coefficients $\mathcal{L}_{0}$ satisfy the assumptions in Theorem 3.2, so the estimate (14) is available. Let $d_{1}=1$ (especially, we set $\mathbf{x}_{1}=x^{1}$ ) and $d_{2}=d-1$. For $u \in C_{\mathrm{loc}}^{\infty}\left(\mathbb{R}^{d+1}\right)$, we take an appropriate $\lambda>0$ and apply the estimate (14) with $T=0$ to $\eta_{m} u$. Then

$$
\begin{aligned}
\mathcal{A}_{m}:=\left\|\eta_{m} u\right\|_{W_{q, p}^{1,2}\left((0, \infty) \times \mathbb{R}^{d}\right)} & \leq N\left\|\left(\mathcal{L}_{0}-\lambda\right)\left(\eta_{m} u\right)\right\|_{L_{q, p}\left((0, \infty) \times \mathbb{R}^{d}\right)} \\
& \leq N \mathcal{B}+N\left\|\eta_{m x} u_{x}\right\|_{L_{q, p}\left((0, \infty) \times \mathbb{R}^{d}\right)}+N \frac{2^{2 m}}{(R-r)^{2}} \mathcal{C},
\end{aligned}
$$

where $N=N(d, p, q, \delta)$ and

$$
\mathcal{B}:=\left\|\left(\mathcal{L}_{0}-\lambda\right) u\right\|_{L_{q, p}\left(\Lambda_{R}\right)}, \quad \mathcal{C}:=\|u\|_{L_{q, p}\left(\Lambda_{R}\right)} .
$$

Observe that, for arbitrary $\varepsilon>0$,

$$
\begin{gathered}
\left\|\eta_{m x} u_{x}\right\|_{L_{q, p}\left((0, \infty) \times \mathbb{R}^{d}\right)}=\left\|\eta_{m x}\left(\eta_{m+1} u\right)_{x}\right\|_{L_{q, p}\left((0, \infty) \times \mathbb{R}^{d}\right)} \\
\leq N \frac{2^{m}}{R-r}\left\|\left(\eta_{m+1} u\right)_{x}\right\|_{L_{q, p}\left((0, \infty) \times \mathbb{R}^{d}\right)} \leq \varepsilon \mathcal{A}_{m+1}+N \varepsilon^{-1} \frac{2^{2 m}}{(R-r)^{2}} \mathcal{C},
\end{gathered}
$$

where the last inequality is due to the interpolation inequality. Thus, (15) yields

$$
\mathcal{A}_{m} \leq N \mathcal{B}+\varepsilon \mathcal{A}_{m+1}+N \varepsilon^{-1} \frac{2^{2 m}}{(R-r)^{2}} \mathcal{C} .
$$


Set $\varepsilon=1 / 8$. Then by multiplying both sides by $\varepsilon$ and summing up, we get

$$
\mathcal{A}_{0}+\sum_{m=1}^{\infty} \varepsilon^{m} \mathcal{A}_{m} \leq N \mathcal{B}+\sum_{m=1}^{\infty} \varepsilon^{m} \mathcal{A}_{m}+N \frac{1}{(R-r)^{2}} \mathcal{C} .
$$

Indeed, the series of $\varepsilon^{m} \mathcal{A}_{m}=8^{-m} \mathcal{A}_{m}$ converges since

$$
\mathcal{A}_{m} \leq N 2^{2 m}(R-r)^{-2}\|u\|_{W_{q, p}^{1,2}\left(\Lambda_{R}\right)} .
$$

Therefore, after taking care of similar terms we see that $\mathcal{A}_{0}$ is less than or equal to the right-hand side of the inequality in the lemma. The left-hand side is obviously less than $\mathcal{A}_{0}$, so the lemma is proved.

Denote

$$
\mathfrak{q}_{r}\left(t, x^{1}\right):=\left(t, t+r^{2}\right) \times\left(x^{1}-r, x^{1}+r\right) \subset \mathbb{R} \times \mathbb{R} .
$$

Especially, $\mathfrak{q}_{r}=\mathfrak{q}_{r}(0,0)=\left(0, r^{2}\right) \times(-r, r)$.

LEMma 5.4. Let $p, q \in[2, \infty)$ be such that $q \geq p$ and $1 / q \geq 1 / p-1 / 3$. Also let $0<r<R<\infty$ and $\gamma=\left(\gamma^{1}, \cdots, \gamma^{d}\right)$ be a multi-index such that $\gamma^{1}=0$. If $v \in C_{\text {loc }}^{\infty}\left(\mathbb{R}^{d+1}\right)$ is a function such that $\mathfrak{L}_{0} v=0$ in $\Lambda_{R}$, then

$$
\left\|D_{x^{\prime}}^{\gamma} v_{t}\right\|_{L_{q, p}\left(\Lambda_{r}\right)}+\sum_{m=0}^{2}\left\|D_{x^{\prime}}^{\gamma} D_{x^{1}}^{m} v\right\|_{L_{q, p}\left(\Lambda_{r}\right)} \leq N\|v\|_{L_{p}\left(\Lambda_{R}\right)},
$$

where $N=N\left(d_{1}, d_{2}, p, q, \delta, \gamma, r, R\right)$.

Proof. It is enough to prove

$$
\|v\|_{L_{q, p}\left(\Lambda_{r}\right)}+\left\|v_{x^{1}}\right\|_{L_{q, p}\left(\Lambda_{r}\right)}+\left\|v_{x^{1} x^{1}}\right\|_{L_{q, p}\left(\Lambda_{r}\right)}+\left\|v_{t}\right\|_{L_{q, p}\left(\Lambda_{r}\right)} \leq N\|v\|_{L_{p}\left(\Lambda_{r_{1}}\right)},
$$

where $r_{1}$ is a number such that $r<r_{1}<R$. To see this, using the fact that $D_{x^{\prime}}^{\gamma} v$ satisfies $\mathfrak{L}_{0} D_{x^{\prime}}^{\gamma} v=0$ in $\Lambda_{R}$, we have the above inequality with $D_{x^{\prime}}^{\gamma} v$ in place of $v$. Then using Lemma 5.2 as many times as needed, we arrive at the desired inequality in the lemma.

If $p=q$, the inequality (16) follows directly from Lemma 5.2, so we consider the case $q>p$. Since $\mathfrak{L}_{0} v=0$ in $\Lambda_{R}$, we have

$$
v_{t}+a^{11}\left(x^{1}\right) v_{x^{1} x^{1}}+\Delta_{d-1} v=\Delta_{d-1} v-\sum_{i \neq 1 \text { or } j \neq 1} a^{i j} v_{x^{i} x^{j}} \text { in } \Lambda_{R} .
$$

If $\tau$ is a number between $r$ and $r_{1}$, by Lemma 5.3, we have

$$
\begin{aligned}
\|v\|_{L_{q, p}\left(\Lambda_{r}\right)} & +\left\|v_{x^{1}}\right\|_{L_{q, p}\left(\Lambda_{r}\right)}+\left\|v_{x^{1} x^{1}}\right\|_{L_{q, p}\left(\Lambda_{r}\right)}+\left\|v_{t}\right\|_{L_{q, p}\left(\Lambda_{r}\right)} \\
& \leq N\left(\left\|v_{x x^{\prime}}\right\|_{L_{q, p}\left(\Lambda_{\tau}\right)}+\|v\|_{L_{q, p}\left(\Lambda_{\tau}\right)}\right),
\end{aligned}
$$

where $N=N(d, p, q, r, \tau, \delta)$. Thus we obtain the inequality (16) once we prove

$$
\left\|v_{x x^{\prime}}\right\|_{L_{q, p}\left(\Lambda_{\tau}\right)}+\|v\|_{L_{q, p}\left(\Lambda_{\tau}\right)} \leq N\|v\|_{L_{p}\left(\Lambda_{r_{1}}\right)} .
$$

However, we prove

$$
\left\|v_{x x^{\prime}}\right\|_{L_{p}^{x^{\prime}} L_{q}^{t, x^{1}}\left(\Lambda_{\tau}\right)}+\|v\|_{L_{p}^{x^{\prime}} L_{q}^{t, x^{1}}\left(\Lambda_{\tau}\right)} \leq N\|v\|_{L_{p}\left(\Lambda_{r_{1}}\right)}
$$


because by Minkowski's inequality (note that $q / p>1$ ), for example,

$$
\left\|v_{x x^{\prime}}\right\|_{L_{q, p}\left(\Lambda_{\tau}\right)}=\left\|v_{x x^{\prime}}\right\|_{L_{q}^{t, x^{1}} L_{p}^{x^{\prime}}\left(\Lambda_{\tau}\right)} \leq\left\|v_{x x^{\prime}}\right\|_{L_{p}^{x^{\prime}} L_{q}^{t, x^{1}}\left(\Lambda_{\tau}\right)} .
$$

To prove (17), for each $x^{\prime} \in B_{R}^{\prime}$, we view $v\left(t, x^{1}, x^{\prime}\right)$ as a function of $\left(t, x^{1}\right)$. By the Sobolev embedding theorem (Lemma 3.3 in Chapter $2[16]$ )

$$
\left\|v\left(\cdot, x^{\prime}\right)\right\|_{L_{q}\left(\mathfrak{q}_{\tau}\right)}+\left\|v_{x^{1}}\left(\cdot, x^{\prime}\right)\right\|_{L_{q}\left(\mathfrak{q}_{\tau}\right)} \leq N\left\|v\left(\cdot, x^{\prime}\right)\right\|_{W_{p}^{1,2}\left(\mathfrak{q}_{\tau}\right)},
$$

where $N=N(p, q, \tau)$. In the above inequality we can replace $v\left(t, x^{1}, x^{\prime}\right)$ by $v_{x^{\prime}}\left(t, x^{1}, x^{\prime}\right)$ or $v_{x^{\prime} x^{\prime}}\left(t, x^{1}, x^{\prime}\right)$ as functions of $\left(t, x^{1}\right)$. Then, for each $x^{\prime} \in B_{R}^{\prime}$, we have

$$
\left\|v\left(\cdot, x^{\prime}\right)\right\|_{L_{q}\left(\mathfrak{q}_{\tau}\right)}+\left\|v_{x x^{\prime}}\left(\cdot, x^{\prime}\right)\right\|_{L_{q}\left(\mathfrak{q}_{\tau}\right)} \leq N \sum_{m=0}^{2}\left\|D_{x^{\prime}}^{m} v\left(\cdot, x^{\prime}\right)\right\|_{W_{p}^{1,2}\left(\mathfrak{q}_{\tau}\right)},
$$

where $D_{x^{\prime}}^{m} v, m=0,1,2$, is $v, v_{x^{\prime}}$, and $v_{x^{\prime} x^{\prime}}$, respectively. By integrating $p$ th power of both sides of the above inequality over $B_{\tau}^{\prime}$ with respect to $x^{\prime} \in \mathbb{R}^{d-1}$, we get

$$
\begin{aligned}
& \|v\|_{L_{p}^{x^{\prime}} L_{q}^{t, x^{1}}\left(\Lambda_{\tau}\right)}+\left\|v_{x x^{\prime}}\right\|_{L_{p}^{x^{\prime}} L_{q}^{t, x^{1}}\left(\Lambda_{\tau}\right)} \leq N \sum_{m=0}^{2} \int_{B_{\tau}^{\prime}}\left\|D_{x^{\prime}}^{m} v\left(\cdot, x^{\prime}\right)\right\|_{W_{p}^{1,2}\left(\mathfrak{q}_{\tau}\right)}^{p} d x^{\prime} \\
& \leq N\left(\sum_{m=0}^{2}\left\|D_{x^{\prime}}^{m} v_{t}\right\|_{L_{p}\left(\Lambda_{\tau}\right)}^{p}+\sum_{l, m=0}^{2}\left\|D_{x^{1}}^{l} D_{x^{\prime}}^{m}\right\|_{L_{p}\left(\Lambda_{\tau}\right)}^{p}\right) \leq N\|v\|_{L_{p}\left(\Lambda_{r_{1}}\right)}^{p},
\end{aligned}
$$

where the last inequality is due to the fact $\mathfrak{L}_{0} v=0$ in $\Lambda_{R}$ and repeated use of Lemma 5.2 . Hence the inequality (17) is proved, and so is the lemma.

Below we use the following notations $(0<\mu<1)$.

$$
|f|_{0, Q_{r}}:=\sup _{(t, x) \in Q_{r}}|f(t, x)|, \quad[f]_{\mu, Q_{r}}:=\sup _{\substack{(t, x),(s, y) \in Q_{r} \\(t, x) \neq(s, y)}} \frac{|f(t, x)-f(s, y)|}{|t-s|^{\mu / 2}+|x-y|^{\mu}} .
$$

Lemma 5.5. Let $p \geq 2$. Assume that $v \in C_{l o c}^{\infty}\left(\mathbb{R}^{d+1}\right)$ is a function such that $\mathfrak{L}_{0} v=0$ in $Q_{4}$. Then

$$
\left[v_{x x^{\prime}}\right]_{\mu, Q_{1}} \leq N\left(d_{1}, d_{2}, p, \delta\right)\|v\|_{L_{p}\left(Q_{4}\right)},
$$

where $\mu=\mu(p) \in(0,1)$.

Proof. We prove

$$
[v]_{\mu, Q_{1}}+\left[v_{x^{1}}\right]_{\mu, Q_{1}} \leq N\|v\|_{L_{p}\left(\Lambda_{\tau}\right)},
$$

where $2<\tau<\sqrt{8}$. If this is done, we can finish the proof using Lemma 5.4 (when $p=q$ ) and the fact that $\mathfrak{L} v_{x^{\prime}}=\mathfrak{L} v_{x^{\prime} x^{\prime}}=0$ in $Q_{4}$ (also note that $\Lambda_{\sqrt{8}} \subset Q_{4}$ ).

Fix a number $q$ as follows. If $p>3$, then $q=p$. If $2 \leq p \leq 3$, then $q$ is a number such that $3<q \leq 6$. We see that $q \geq p$ and $1 / q \geq 1 / p-1 / 3$. 
We first prove

$$
\max _{x^{\prime} \in B_{1}^{\prime}}\left\|v\left(\cdot, x^{\prime}\right)\right\|_{W_{q}^{1,2}\left(\mathfrak{q}_{1}\right)}+\max _{x^{\prime} \in B_{1}^{\prime}}\left\|v_{x^{\prime}}\left(\cdot, x^{\prime}\right)\right\|_{W_{q}^{1,2}\left(\mathfrak{q}_{1}\right)} \leq N\|v\|_{L_{p}\left(Q_{\tau}\right)} .
$$

Consider $v\left(t, x^{1}, x^{\prime}\right)$ as a function of $x^{\prime} \in B_{1}^{\prime}$. By the Sobolev embedding theorem there exist $N$ and $k$ such that, for each $\left(t, x^{1}\right) \in \mathfrak{q}_{1}$,

$$
\max _{x^{\prime} \in B_{1}^{\prime}}\left|v\left(t, x^{1}, x^{\prime}\right)\right| \leq N\left\|v\left(t, x^{1}, \cdot\right)\right\|_{W_{p}^{k}\left(B_{1}^{\prime}\right)},
$$

where $\left\|v\left(t, x^{1}, \cdot\right)\right\|_{W_{p}^{k}\left(B_{1}^{\prime}\right)}$ is the $W_{p}^{k}\left(B_{1}^{\prime}\right)$ norm of $v$ as a function of $x^{\prime}$. Similarly, by considering $v_{t}\left(t, x^{1}, x^{\prime}\right)$ as well as $D_{x^{1}}^{m} v\left(t, x^{1}, x^{\prime}\right), m=1,2$, as functions of $x^{\prime} \in B_{1}^{\prime}$, we have the same inequalities as above with $v_{t}$ or $D_{x^{1}}^{m} v$ in place of $v$. Thus, for each $\left(t, x^{1}\right) \in \mathfrak{q}_{1}$,

$$
\begin{gathered}
\max _{x^{\prime} \in B_{1}^{\prime}}\left|v_{t}\left(t, x^{1}, x^{\prime}\right)\right|+\sum_{m=0}^{2} \max _{x^{\prime} \in B_{1}^{\prime}}\left|D_{x^{1}}^{m} v\left(t, x^{1}, x^{\prime}\right)\right| \\
\leq N\left(\left\|v_{t}\left(t, x^{1}, \cdot\right)\right\|_{W_{p}^{k}\left(B_{1}^{\prime}\right)}+\sum_{m=0}^{2}\left\|D_{x^{1}}^{m} v\left(t, x^{1}, \cdot\right)\right\|_{W_{p}^{k}\left(B_{1}^{\prime}\right)}\right) .
\end{gathered}
$$

This implies that

$$
\begin{gathered}
\max _{x^{\prime} \in B_{1}^{\prime}}\left\|v\left(\cdot, x^{\prime}\right)\right\|_{W_{q}^{1,2}\left(\mathfrak{q}_{1}\right)} \\
\leq N\left(\int_{\mathfrak{q}_{1}}\left\|v_{t}\left(t, x^{1}, \cdot\right)\right\|_{W_{p}^{k}\left(B_{1}^{\prime}\right)}^{q}+\sum_{m=0}^{2}\left\|D_{x^{1}}^{m} v\left(t, x^{1}, \cdot\right)\right\|_{W_{p}^{k}\left(B_{1}^{\prime}\right)}^{q} d x^{1} d t\right)^{1 / q} \\
\leq N \sum_{|\gamma| \leq k}\left(\left\|D_{x^{\prime}}^{\gamma} v_{t}\right\|_{L_{q, p}\left(\Lambda_{1}\right)}+\sum_{m=0}^{2}\left\|D_{x^{\prime}}^{\gamma} D_{x^{1}}^{m} v\right\|_{L_{q, p}\left(\Lambda_{1}\right)}\right) \leq N\|v\|_{L_{p}\left(\Lambda_{\tau}\right)},
\end{gathered}
$$

where the last inequality is from Lemma 5.4. Thus the first term in (19) is proved to be less than or equal to the right hand side of the inequality. To complete the inequality (19), we repeat the same argument as above with $v_{x^{\prime}}$ in place of $v$.

Now we obtain the following inequalities. For each $x^{\prime} \in B_{1}^{\prime}$, view $v\left(t, x^{1}, x^{\prime}\right)$ and $v_{x^{\prime}}\left(t, x^{1}, x^{\prime}\right)$ as functions of $\left(t, x^{1}\right)$ defined on $\mathfrak{q}_{1}$. Then by the embedding theorem (Lemma 3.3 in Chapter $2[16]$ )

$$
\begin{aligned}
\left|v_{x^{\prime}}\left(\cdot, x^{\prime}\right)\right|_{0, \mathfrak{q}_{1}}+\left|v_{x^{1} x^{\prime}}\left(\cdot, x^{\prime}\right)\right|_{0, \mathfrak{q}_{1}} & \leq N\left\|v_{x^{\prime}}\left(\cdot, x^{\prime}\right)\right\|_{W_{q}^{1,2}\left(\mathfrak{q}_{1}\right)}, \\
{\left[v\left(\cdot, x^{\prime}\right)\right]_{\mu, \mathfrak{q}_{1}}+\left[v_{x^{1}}\left(\cdot, x^{\prime}\right)\right]_{\mu, \mathfrak{q}_{1}} } & \leq N\left\|v\left(\cdot, x^{\prime}\right)\right\|_{W_{q}^{1,2}\left(\mathfrak{q}_{1}\right)}
\end{aligned}
$$

for each $x^{\prime} \in B_{1}^{\prime}$, where $\mu=1-3 / q$ and $N=N(q)$.

Finally, we prove the inequality (18). Note that

$$
\left|v_{x^{1}}(t, x)-v_{x^{1}}(s, y)\right| \leq I_{1}+I_{2},
$$


where

$$
I_{1}=\left|v_{x^{1}}\left(t, x^{1}, x^{\prime}\right)-v_{x^{1}}\left(s, y^{1}, x^{\prime}\right)\right|, \quad I_{2}=\left|v_{x^{1}}\left(s, y^{1}, x^{\prime}\right)-v_{x^{1}}\left(s, y^{1}, y^{\prime}\right)\right| .
$$

By (21) and (19)

$$
I_{1} \leq N\left(|t-s|^{\mu / 2}+\left|x^{1}-y^{1}\right|^{\mu}\right)\|v\|_{L_{p}\left(\Lambda_{\tau}\right)} .
$$

To take care of $I_{2}$, we use (20) and (19).

$$
\begin{gathered}
I_{2} \leq\left|x^{\prime}-y^{\prime}\right| \max _{z^{\prime} \in B_{1}^{\prime}}\left|v_{x^{1} x^{\prime}}\left(s, y^{1}, z^{\prime}\right)\right| \leq\left|x^{\prime}-y^{\prime}\right| \max _{z^{\prime} \in B_{1}^{\prime}}\left\|v_{x^{\prime}}\left(\cdot, z^{\prime}\right)\right\|_{W_{q}^{1,2}\left(\mathfrak{q}_{1}\right)} \\
\leq\left|x^{\prime}-y^{\prime}\right|\|v\|_{L_{p}\left(\Lambda_{\tau}\right)} .
\end{gathered}
$$

This shows that

$$
\left[v_{x^{1}}\right]_{\mu, \nu, Q_{1}} \leq N\|v\|_{L_{p}\left(Q_{\tau}\right)} .
$$

By repeating the above argument with $v$ in place of $v_{x^{1}}$, we complete the proof of the inequality (18). The lemma is proved.

Lemmas similar to Lemma 3.7 and 3.8 in section 3 are repeated below, but since the operator $\mathfrak{L}_{\lambda}$ is being dealt with, the lemmas have to be modified as follows.

Lemma 5.6. Let $p \geq 2$ and $\lambda \geq 0$. For every $v \in C_{\text {loc }}^{\infty}\left(\mathbb{R}^{d+1}\right)$ such that $\mathfrak{L}_{\lambda} v=0$ in $Q_{4}$, we have

$$
\left[v_{x x^{\prime}}\right]_{\mu, Q_{1}} \leq N\left(\left\|v_{x x}\right\|_{L_{p}\left(Q_{4}\right)}+\left\|v_{t}\right\|_{L_{p}\left(Q_{4}\right)}+\sqrt{\lambda}\left\|v_{x}\right\|_{L_{p}\left(Q_{4}\right)}\right),
$$

where $\mu=\mu(p) \in(0,1)$ and $N=N(d, p, \delta)$.

Proof. We follow the steps in the proof of Lemma 3.7, but the sup-norms of the derivatives of $v$ on $Q_{1}$ have to be replaced by $\left[v_{x x^{\prime}}\right]_{\mu, Q_{1}}$.

Lemma 5.7. Let $p \geq 2, \lambda \geq 0, \kappa \geq 4$, and $r \in(0, \infty)$. Let $v \in C_{l o c}^{\infty}\left(\mathbb{R}^{d+1}\right)$ be such that $\mathfrak{L}_{\lambda} v=0$ in $Q_{\kappa r}$. Then there is a constant $N$, depending only on $d, p$, and $\delta$, such that

$$
f_{Q_{r}}\left|v_{x x^{\prime}}(t, x)-\left(v_{x x^{\prime}}\right)_{Q_{r}}\right|^{p} d x d t \leq N \kappa^{-\mu p}\left(\left|v_{x x}\right|^{p}+\left|v_{t}\right|^{p}+\lambda^{p / 2}\left|v_{x}\right|^{p}\right)_{Q_{\kappa r}},
$$

where $\mu=\mu(p) \in(0,1)$.

Proof. We use Lemma 5.6. Note that

$$
f_{Q_{1}}\left|v_{x x^{\prime}}(t, x)-\left(v_{x x^{\prime}}\right)_{Q_{1}}\right|^{p} d x d t \leq N\left[v_{x x^{\prime}}\right]_{\mu, Q_{1}}^{p}
$$

and

$$
\left[\check{v}_{x x^{\prime}}\right]_{\mu, Q_{1}}=\left(\frac{\kappa}{4}\right)^{\mu+2}\left[v_{x x^{\prime}}\right]_{\mu, Q_{\kappa / 4}} \quad \text { if } \quad \check{v}(t, x)=v\left(\left(\frac{\kappa}{4}\right)^{2} t, \frac{\kappa}{4} x\right) .
$$

Using these as well as the argument in the proof of Lemma 3.8, one can complete the proof. $\mathrm{Q}$ 
Now we arrive at the following theorem, the proof of which is almost identical to that of Theorem 3.9.

THEOREM 5.8. Let $p \geq 2$. Then there is a constant $N$, depending only on $d, p$, and $\delta$, such that, for any $u \in W_{p}^{1,2}\left(\mathbb{R}^{d+1}\right), r \in(0, \infty)$, and $\kappa \geq 8$,

$$
f_{Q_{r}}\left|u_{x x^{\prime}}(t, x)-\left(u_{x x^{\prime}}\right)_{Q_{r}}\right|^{p} d x d t \leq N \kappa^{d+2}\left(\left|\mathfrak{L}_{0} u\right|^{p}\right)_{Q_{\kappa r}}+N \kappa^{-\mu p}\left(\left|u_{x x}\right|^{p}\right)_{Q_{\kappa r}},
$$

where $\mu=\mu(p) \in(0,1)$.

6. Proof of Theorem 2.3 and 2.4. In this section, as stated in Theorem 2.3, we deal with coefficients of $L$ satisfying Assumption 2.1 and 2.2. Also note that the coefficients $a^{i j}(t, x)$ are independent of $x^{\prime} \in \mathbb{R}^{d-1}$ if $p=2$. Throughout the section, we denote

$$
L_{0} u=u_{t}+a^{i j}(t, x) u_{x^{i} x^{j}} .
$$

As noted earlier, due to Theorem 3.2 in this paper, the results in [7] are now available. This implies that, by the same reasoning as in the proof of Lemma 4.1, the inequalities in Lemma 4.1 are possible with $L_{0}$ defined above. Then using the results in section 5 and repeating the proof of Theorem 4.2 (with necessary changes), we obtain

THEOREM 6.1. Let $p \geq 2$. In case $p=2$, we assume that the coefficients $a^{i j}(t, x)$ of $L_{0}$ are independent of $x^{\prime} \in \mathbb{R}^{d-1}$. Then there exists a constant $N$, depending on $d$, $p$, $\delta$, and the function $\omega$, such that, for any $u \in C_{0}^{\infty}\left(\mathbb{R}^{d+1}\right), \kappa \geq 16$, and $r \in(0,1 / \kappa]$, we have

$$
\begin{gathered}
f_{Q_{r}}\left|u_{x x^{\prime}}(t, x)-\left(u_{x x^{\prime}}\right)_{Q_{r}}\right|^{p} d x d t \\
\leq N \kappa^{d+2}\left(\left|L_{0} u\right|^{p}\right)_{Q_{\kappa r}}+N\left(\kappa^{-\mu p}+\kappa^{d+2}\left(a_{\kappa r}^{\#}\right)^{1 / 2}\right)\left(\left|u_{x x}\right|^{p}\right)_{Q_{\kappa r}},
\end{gathered}
$$

where $\mu=\mu(p) \in(0,1)$.

In the following we state two lemmas corresponding to Lemma 4.4 and Lemma 4.5 , respectively. Since we are dealing with $a^{i j}$ different from those in section 4 , we have different statements, but the proofs are almost the same as those for Lemma 4.4 and Lemma 4.5.

Lemma 6.2. Let $p \geq 2$. In case $p=2$, we assume that the coefficients $a^{i j}(t, x)$ of $L_{0}$ are independent of $x^{\prime} \in \mathbb{R}^{d-1}$. Then there exists a constant $N$, depending on $d_{1}, d_{2}, p, \delta$, and the function $\omega$, such that, for any $u \in C_{0}^{\infty}\left(\mathbb{R}^{d+1}\right), \kappa \geq 16 \sqrt{2}$, and $r \in(0,1 / \kappa]$, we have

$$
\begin{gathered}
f_{Q_{r}^{d_{1}}} f_{Q_{r}^{d_{1}}}\left|\varphi\left(t, \mathbf{x}_{1}\right)-\varphi\left(s, \mathbf{y}_{1}\right)\right|^{p} d \mathbf{x}_{1} d t d \mathbf{y}_{1} d s \\
\leq N \kappa^{d+2}\left(\psi^{p}\right)_{Q_{\kappa r}^{d_{1}}}+N\left(\kappa^{-\mu p}+\kappa^{d+2}\left(a_{\kappa r}^{\#}\right)^{1 / 2}\right)\left(\zeta^{p}\right)_{Q_{\kappa r}^{d_{1}}},
\end{gathered}
$$


where $\mu=\mu(p) \in(0,1)$,

$$
\begin{gathered}
\varphi\left(t, \mathbf{x}_{1}\right)=\left\|u_{x x^{\prime}}\left(t, \mathbf{x}_{1}, \cdot\right)\right\|_{p\left(d_{2}\right)}, \\
\zeta\left(t, \mathbf{x}_{1}\right)=\left\|u_{x x}\left(t, \mathbf{x}_{1}, \cdot\right)\right\|_{p\left(d_{2}\right)}, \quad \psi\left(t, \mathbf{x}_{1}\right)=\left\|L_{0} u\left(t, \mathbf{x}_{1}, \cdot\right)\right\|_{p\left(d_{2}\right)} .
\end{gathered}
$$

Lemma 6.3. Let $p \geq 2$. In case $p=2$, we assume that the coefficients $a^{i j}(t, x)$ of $L_{0}$ are independent of $x^{\prime} \in \mathbb{R}^{d-1}$. Let $R \in(0,1]$ and $u$ be a function in $C_{0}^{\infty}\left(\mathbb{R}^{d+1}\right)$ such that

$$
u(t, x)=u\left(t, \mathbf{x}_{1}, \mathbf{x}_{2}\right)=0 \quad \text { if }\left(t, \mathbf{x}_{1}\right) \notin\left(0, R^{4}\right) \times B_{R^{2}}^{d_{1}} .
$$

Then

$$
\begin{gathered}
\varphi^{\#}\left(t, \mathbf{x}_{1}\right) \leq N \kappa^{(d+2) / p}\left(M \psi^{p}\left(t, \mathbf{x}_{1}\right)\right)^{1 / p} \\
+N\left((\kappa R)^{\left(d_{1}+2\right)(1-1 / p)}+\kappa^{-\mu}+\kappa^{(d+2) / p}(\omega(R))^{1 / 2 p}\right)\left(M \zeta^{p}\left(t, \mathbf{x}_{1}\right)\right)^{1 / p}
\end{gathered}
$$

for all $\kappa \geq 16 \sqrt{2}$ and $\left(t, \mathbf{x}_{1}\right) \in \mathbb{R} \times \mathbb{R}^{d_{1}}$, where $\mu=\mu(p) \in(0,1), N=N\left(d_{1}, d_{2}, p, \delta, \omega\right)$, and the functions $\varphi, \zeta, \psi$ are those defined as in Lemma 6.2.

The proof of the next corollary clearly shows the necessity of the result for the case with $a^{i j}(t, x)$ measurable in $x^{1} \in \mathbb{R}$ and VMO in $\left(t, x^{\prime}\right) \in \mathbb{R}^{d}$ (Theorem 3.2, specifically, Corollary 4.6). As in corollary 4.6, we set $L_{q, p}=L_{q}^{t, \mathbf{x}_{1}} L_{p}^{\mathbf{x}_{2}}\left(\mathbb{R} \times \mathbb{R}^{d}\right)$. However, as we see in Remark 4.7, the result holds true as well for $L_{q, p}=L_{q}^{\mathbf{x}_{1}} L_{p}^{t, \mathbf{x}_{2}}\left(\mathbb{R} \times \mathbb{R}^{d}\right)$.

Corollary 6.4. Let $q>p \geq 2$. Assume that, in case $p=2$, the coefficients $a^{i j}$ of $L_{0}$ are independent of $x^{\prime} \in \mathbb{R}^{d-1}$. Then there exist $R$ and $N$, depending only on $d_{1}, d_{2}, p, q, \delta$, and the function $\omega$, such that

$$
\left\|u_{t}\right\|_{L_{q, p}}+\left\|u_{x x}\right\|_{L_{q, p}} \leq N\left\|L_{0} u\right\|_{L_{q, p}}
$$

for any $u \in C_{0}^{\infty}\left(\mathbb{R}^{d+1}\right)$ satisfying

$$
u(t, x)=u\left(t, \mathbf{x}_{1}, \mathbf{x}_{2}\right)=0 \quad \text { if }\left(t, \mathbf{x}_{1}\right) \notin\left(0, R^{4}\right) \times B_{R^{2}}^{d_{1}} .
$$

Proof. As is seen in Corollary 4.6, from Lemma 6.3, we obtain

$$
\begin{gathered}
\left\|u_{x x^{\prime}}\right\|_{L_{q, p}} \leq N \kappa^{(d+2) / p}\left\|L_{0} u\right\|_{L_{q, p}} \\
+N\left((\kappa R)^{\left(d_{1}+2\right)(1-1 / p)}+\kappa^{-\mu}+\kappa^{(d+2) / p}(\omega(R))^{1 / 2 p}\right)\left\|u_{x x}\right\|_{L_{q, p}}
\end{gathered}
$$

for all $\kappa \geq 16 \sqrt{2}$ and $R \in(0,1]$. To obtain an estimate for $u_{x^{1} x^{1}}$, we set

$$
g=L_{0} u+\Delta_{d-1} u-\sum_{i \neq 1, j \neq 1} a^{i j} u_{x^{i} x^{j}}
$$

where $\Delta_{d-1} u=\sum_{i=2}^{d} u_{x^{i} x^{i}}$. Then

$$
L_{1} u:=u_{t}+a^{11} u_{x^{1} x^{1}}+\Delta_{d-1} u=g
$$


and the operator $L_{1}$ satisfies the assumptions in Corollary 4.6. Thus there exist $R_{1}=R_{1}\left(d_{1}, d_{2}, p, q, \delta, \omega\right)$ and $N=N\left(d_{1}, d_{2}, q, \delta, \omega\right)$ such that

$$
\left\|u_{x^{1} x^{1}}\right\|_{L_{q, p}} \leq N\|g\|_{L_{q, p}} \leq N\left(\left\|L_{0} u\right\|_{L_{q, p}}+\left\|u_{x x^{\prime}}\right\|_{L_{q, p}}\right)
$$

for all $u \in C_{0}^{\infty}\left(\mathbb{R}^{d+1}\right)$ such that

$$
u(t, x)=u\left(t, \mathbf{x}_{1}, \mathbf{x}_{2}\right)=0 \quad \text { if }\left(t, \mathbf{x}_{1}\right) \notin\left(0, R_{1}^{4}\right) \times B_{R_{1}^{2}}^{d_{1}} .
$$

From this together with the estimate for $\left\|u_{x x^{\prime}}\right\|_{L_{q, p}}$ above, we have

$$
\begin{gathered}
\left\|u_{x x}\right\|_{L_{q, p}} \leq N \kappa^{(d+2) / p}\left\|L_{0} u\right\|_{L_{q, p}} \\
+N\left((\kappa R)^{\left(d_{1}+2\right)(1-1 / p)}+\kappa^{-\mu}+\kappa^{(d+2) / p}(\omega(R))^{1 / 2 p}\right)\left\|u_{x x}\right\|_{L_{q, p}}
\end{gathered}
$$

if $u(t, x)=u\left(t, \mathbf{x}_{1}, \mathbf{x}_{2}\right)=0$ for $\left(t, \mathbf{x}_{1}\right) \notin\left(0, R^{4}\right) \times B_{R^{2}}^{d_{1}} \cap\left(0, R_{1}^{4}\right) \times B_{R_{1}^{2}}^{d_{1}}$. Now we choose a large $\kappa$ and then a small $R$ (smaller than $R_{1}$ ) such that

$$
N\left((\kappa R)^{\left(d_{1}+1\right)(1-1 / p)}+\kappa^{-\mu}+\kappa^{(d+2) / p}(\omega(R))^{1 / 2 p}\right)<1 / 2
$$

(note that $\mu>0$ ). Then we have

$$
\left\|u_{x x}\right\|_{L_{q, p}} \leq 2 N \kappa^{(d+2) / p}\left\|L_{0} u\right\|_{L_{q, p}} .
$$

Finally, notice that

$$
\left\|u_{t}\right\|_{L_{q, p}}=\left\|L_{0} u-a^{i j} u_{x^{i} x^{j}}\right\|_{L_{q, p}} \leq\left\|L_{0} u\right\|_{L_{q, p}}+N\left\|u_{x x}\right\|_{L_{q, p}} .
$$

The corollary is now proved.

As Corollary 4.6 implies Theorem 4.8, the above corollary implies the following theorem.

THEOREM 6.5. Under the assumptions on $L$ given in Theorem 2.3, there exist $\lambda_{0} \geq 0$ and $N$, depending only on $d_{1}, d_{2}, \delta, K, p, q$, and the function $\omega$, such that

$$
\begin{gathered}
\lambda\|u\|_{L_{q, p}\left((T, \infty) \times \mathbb{R}^{d}\right)}+\sqrt{\lambda}\left\|u_{x}\right\|_{L_{q, p}\left((T, \infty) \times \mathbb{R}^{d}\right)}+\left\|u_{x x}\right\|_{L_{q, p}\left((T, \infty) \times \mathbb{R}^{d}\right)} \\
+\left\|u_{t}\right\|_{L_{q, p}\left((T, \infty) \times \mathbb{R}^{d}\right)} \leq N\|(L-\lambda) u\|_{L_{q, p}\left((T, \infty) \times \mathbb{R}^{d}\right)}
\end{gathered}
$$

for all $\lambda \geq \lambda_{0}$ and $u \in W^{1,2}\left((T, \infty) \times \mathbb{R}^{d}\right)$, where $-\infty \leq T<\infty$.

The following proof illustrate how parabolic results can be used proving the unique solvability of elliptic equations.

Proof of Theorem 2.4. It is enough to prove the a priori estimate (5) for $u \in$ $C_{0}^{\infty}\left(\mathbb{R}^{d}\right)$. Set $w(t, x)=\eta(t / n) u(x)$, where $\eta(t) \in C_{0}^{\infty}(\mathbb{R})$, and $L w=w_{t}+E w$. Since the operator $L$ satisfies the assumptions in Theorem 6.5, there exist $\lambda_{0}$ and $N$ such that

$$
\lambda\|w\|_{L_{q, p}\left(\mathbb{R}^{d+1}\right)}+\sqrt{\lambda}\left\|w_{x}\right\|_{L_{q, p}\left(\mathbb{R}^{d+1}\right)}+\left\|w_{x x}\right\|_{L_{q, p}\left(\mathbb{R}^{d+1}\right)}
$$




$$
+\left\|w_{t}\right\|_{L_{q, p}\left(\mathbb{R}^{d+1}\right)} \leq N\|(L-\lambda) w\|_{L_{q, p}\left(\mathbb{R}^{d+1}\right)}
$$

for all $\lambda \geq \lambda_{0}$. Note that

$$
\begin{gathered}
\|w\|_{L_{q, p}\left(\mathbb{R}^{d+1}\right)}=n^{1 / q} c_{1}\|u\|_{L_{q, p}\left(\mathbb{R}^{d}\right)}, \quad\left\|w_{x}\right\|_{L_{q, p}\left(\mathbb{R}^{d+1}\right)}=n^{1 / q} c_{1}\left\|u_{x}\right\|_{L_{q, p}\left(\mathbb{R}^{d}\right)}, \\
\left\|w_{x x}\right\|_{L_{q, p}\left(\mathbb{R}^{d+1}\right)}=n^{1 / q} c_{1}\left\|u_{x x}\right\|_{L_{q, p}\left(\mathbb{R}^{d}\right)}, \\
\|(L-\lambda) w\|_{L_{q, p}\left(\mathbb{R}^{d+1}\right)} \leq n^{1 / q} c_{1}\|E u-\lambda u\|_{L_{q, p}\left(\mathbb{R}^{d}\right)}+n^{1 / q-1} c_{2}\|u\|_{L_{q, p}\left(\mathbb{R}^{d}\right)},
\end{gathered}
$$

where $c_{1}=\|\eta\|_{L_{q}(\mathbb{R})}$ and $c_{2}=\left\|\eta^{\prime}\right\|_{L_{q}(\mathbb{R})}$. Therefore,

$$
\begin{aligned}
& \lambda\|u\|_{L_{q, p}\left(\mathbb{R}^{d}\right)}+\sqrt{\lambda}\left\|u_{x}\right\|_{L_{q, p}\left(\mathbb{R}^{d}\right)}+\left\|u_{x x}\right\|_{L_{q, p}\left(\mathbb{R}^{d}\right)} \\
& \leq N\|E u-\lambda u\|_{L_{q, p}\left(\mathbb{R}^{d}\right)}+N n^{-1}\|u\|_{L_{q, p}\left(\mathbb{R}^{d}\right)} .
\end{aligned}
$$

By letting $n \rightarrow \infty$, we arrive at the desired estimate.

Proof of Theorem 2.3. This theorem is proved in the same manner as Theorem 3.2 using the estimate in Theorem 6.5.

\section{REFERENCES}

[1] M. Bramanti and M. Cristina Cerutti, $W_{p}^{1,2}$ solvability for the Cauchy-Dirichlet problem for parabolic equations with VMO coefficients, Comm. Partial Differential Equations, 18:910 (1993), pp. 1735-1763.

[2] F. Chiarenza, M. Frasca and P. Longo, $W^{2, p}$-solvability of the Dirichlet problem for nondivergence elliptic equations with VMO coefficients, Trans. Amer. Math. Soc., 336:2 (1993), pp. 841-853.

[3] R. Denk, M. Hieber and J. Prüss, R-boundedness, Fourier multipliers and problems of elliptic and parabolic type, Mem. Amer. Math. Soc., 166:788 (2003), pp. viii+114.

[4] Y. Giga And H. SoHr, Abstract $L^{p}$ estimates for the Cauchy problem with applications to the Navier-Stokes equations in exterior domains, J. Funct. Anal., 102:1 (1991), pp. 72-94.

[5] D. Gilbarg and N.S. Trudinger, Elliptic Partial Differential Equations of Second Order, Springer, 2nd edition, 1983.

[6] R. Haller-Dintelmann, H. Heck and M. Hieber, $L^{p}-L^{q}$ estimates for parabolic systems in non-divergence form with VMO coefficients, J. London Math. Soc. (2), 74:3 (2006), pp. $717-736$.

[7] D. KIM, Parabolic equations with measurable coefficients II, J. Math. Anal. Appl., 334:1 (2007), pp. $534-548$.

[8] D. Kim And N.V. Krylov, Elliptic differential equations with coefficients measurable with respect to one variable and VMO with respect to the others, SIAM J. Math. Anal., 39:2 (2007), pp. 489-506.

[9] D. Kim and N.V. Krylov, Parabolic equations with measurable coefficients, Potential Analysis, 26:4 (2007), pp. 345-361.

[10] N.V. KRYLov, The Calderón-Zygmund theorem and its applications to parabolic equations, Algebra i Analiz, 13:4 (2001), pp. 1-25.

[11] N.V. KRYLOV, The heat equation in $L_{q}\left((0, T), L_{p}\right)$-spaces with weights, SIAM J. Math. Anal., 32:5 (2001), pp. 1117-1141 (electronic).

[12] N.V. KrYlov, Parabolic equations in $L_{p}$-spaces with mixed norms, Algebra i Analiz, 14:4 (2002), pp. 91-106.

[13] N.V. KrYLOv, Parabolic and elliptic equations with VMO coefficients, Comm. Partial Differential Equations, 32:3 (2007), pp. 453-475.

[14] N.V. Krylov, Parabolic equations with VMO coefficients in Sobolev spaces with mixed norms, J. Funct. Anal., 250:2 (2007), pp. 521-558. 
[15] N.V. Krylov, Lectures on elliptic and parabolic equations in Sobolev spaces, volume 96 of "Graduate Studies in Mathematics", American Mathematical Society, 2008.

[16] O.A. Ladyženskaja, V.A. Solonnikov, and N.N. Ural'Ceva, Linear and quasilinear equations of parabolic type, translated from the Russian by S. Smith. Translations of Mathematical Monographs, Vol. 23. American Mathematical Society, Providence, R.I., 1967.

[17] G.M. Lieberman, Second order parabolic differential equations, World Scientific Publishing Co. Inc., River Edge, NJ, 1996.

[18] P. Maremonti and V.A. Solonnikov, On estimates for the solutions of the nonstationary Stokes problem in S. L. Sobolev anisotropic spaces with a mixed norm, Zap. Nauchn. Sem. S.-Peterburg. Otdel. Mat. Inst. Steklov. (POMI), 222 (Issled. po Linein. Oper. i Teor. Funktsii. 23), 309 (1995), pp. 124-150, 1995.

[19] A. Maugeri, D.K. Palagachev and L.G. Softova, Elliptic and parabolic equations with discontinuous coefficients, volume 109 of "Mathematical Research", Wiley-VCH Verlag Berlin GmbH, Berlin, 2000.

[20] L.G. Softova And P. Weidemaier, Quasilinear parabolic problem in spaces of maximal regularity, J. Nonlinear Convex Anal., 7:3 (2006), pp. 529-540.

[21] P. Weidemaier, Maximal regularity for parabolic equations with inhomogeneous boundary conditions in Sobolev spaces with mixed $L_{p}$-norm, Electron. Res. Announc. Amer. Math. Soc.,, 8 (2002), pp. 47-51 (electronic). 
